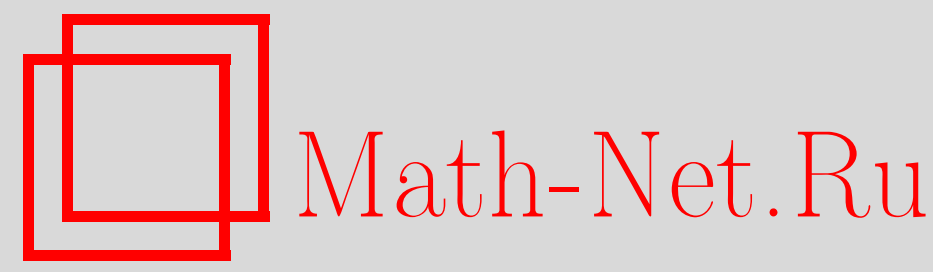

Ю. Г. Прохоров, В. В. Шокуров, Первая основная теорема о дополнениях: от глобального к локальному, Изв. РАН. Сер. матем., 2001, том 65, выпуск 6, 99-128

DOI: https://doi.org/10.4213/im366

Использование Общероссийского математического портала Math-Net.Ru подразумевает, что вы прочитали и согласны с пользовательским соглашением http://www . mathnet.ru/rus/agreement

Параметры загрузки:

IP: 3.80 .181 .102

26 апреля 2023 г., $11: 35: 22$ 
УДК 512.7

\author{
Ю. Г. Прохоров, В.В. Шокуров
}

\title{
Первая основная теорема о дополнениях: от глобального к локальному
}

\begin{abstract}
Цель настоящей статьи - прояснить и обобщить методы работы [24] (см. также [18] и [19]). Установлено, что для локальных стягиваний Фано существование дополнений может быть редуцировано к существованию дополнений для проективных многообразий Фано меньшей размерности.

Библиографиия: 26 наименований.
\end{abstract}

\section{Введение}

Цель настоящей статьи - прояснить и обобшить методы работы $[24, \S 7]$ (см. также [18], [19]). Докажем, что для локальных стягиваний Фано существование дополнений может быть редуцировано к существованию дополнений для проективных многообразий $\Phi$ ано меньшей размерности. Основная гипотеза о $n$-дополнениях (гипотеза 1.3 работы [24]) утверждает, что они ограничены для каждой заданной размерности.

Многочисленные результаты, полученные в работах [1], [6], [7], [9], [10], [14], [19], [21], [23], [24], свидетельствуют в пользу этой гипотезы. По сушеству, $n$-дополнение - это "хороший" элемент в кратной антилогканонической линейной системе. Как было отмечено в [23], дополнения имеют ряд хороших свойств, согласованных с ограничениями линейных систем и с теоремой Каваматы-Фивега об обрашении в нуль. Последнее объясняет нетривиальное свойство-определение $n$-дополнений (cp. (1.1) ниже). В основной гипотезе мы рассмотрим логпары $(X / Z, D)$, состоящие из стягиваний $\Phi$ ано $X / Z$ и гранищ $D$. Чтобы использовать индукцию в доказательстве гипотезы, нам необходимо разделить логпары и их дополнения на два класса по размерности базы $Z$ : локальный, если $\operatorname{dim}(Z)>0$, и глобальньй в противном случае. Эквивалентно, в глобальном случае $Z$ - точка, а $X$ - проективное логмногообразие Фано. Мы докажем сушествование $n$-дополнения для локального логстягивания $\Phi$ ано, где $n \in N$ и множество $N$ определяется классом проективных логмногообразий Фано меньшей размерности. Это называется первой основной теоремой о дополнениях (см. теорему 3.1 ниже): от глобального к локальному случаю. Доказательство использует логарифмическую программу минимальных моделей (логПММ), таким образом, оно является условным для размерностей $n=\operatorname{dim}(X) \geqslant 4$ и полным для $n \leqslant 3$. Основная идея состоит в том, чтобы продолжить $n$-дополнение с центрального слоя хорошей модификации для $(X / Z, D)$

Работа первого автора частично поддержана грантами INTAS-OPEN-97-2072 и РФФИ (грант № 99-01-01132). Работа второго автора выполнена при частичной поддержке гранта NSF-9800807. 
(ср. доказательство теоремы 5.6 и пример 5.2 в [23]). Более того, такой подход позволяет контролировать некоторые численные инварианты дополнений, например индексы, их тип (исключительный или неисключительный) и регулярность (cp. $[24, \S 7])$.

Вторая теорема, от локального к глобальному случаю, будет обсуждаться в следующей статье. Ее прототип представляет собой глобальный случай, рассмотренный в [24], который использует локальные и индуктивные дополнения $[24, \S 2]$ (ср. также понятие “тигра" в [9]). Элементарный, но на самом деле достаточно общий случай второй теоремы - теорема 5.1. Она представляет собой модификацию первой теоремы. Этот шаг также показывает, что главная трудность кроется в гипотезе Борисова-Алексеева (см. гипотезу 1.8 ) и касается $\varepsilon_{d}$-логтерминальных многообразий Фано размерности $d$, а именно что они ограничены для некоторых $\varepsilon_{d}>0$, в зависимости от размерности $d$. В частности, $\varepsilon_{2}=6 / 7$ для размерности 2 .

Статья организована следующим образом: $\S 1$ - вспомогательньй, в 22 вводится очень важное понятие исключительных пар, в $§ 3$ мы доказываем основной результат (теорема 3.1), некоторые следствия и приложения обсуждаются в $\S 4$ и, наконец, в $§ 5$ мы представляем глобальный вариант теоремы 3.1 .

Первый автор выражает искреннюю благодарность Токийскому технологическому институту за гостеприимство и прекрасные условия для работы, предоставленные ему во время визита в 1999-2000 гг., в течение которого была подготовлена эта работа.

\section{§ 1. Предварительные сведения}

Пусть $K(X)$ - поле рациональных функции многообразия $X$; запись $D_{1} \approx D_{2}$ означает, что простые дивизоры задают одно и то же дискретное нормирование поля $K(X) ; K_{X}-$ канонический дивизор (Вейля), мы часто будем писать просто $K$, если это не ведет к разночтениям.

Все рассматриваемые многообразия суть алгебраические и определены над полем комплексных чисел $\mathbb{C}$. Стягивание $f: Y \rightarrow X$-это проективный морфизм нормального многообразия такой, что $f_{*} \mathscr{O}_{Y}=\mathscr{O}_{X}$. Раздутия и сдутия - это бирациональные стягивания. Использованы стандартные определения, сокращения и обозначения из программы минимальных моделей такие, как ПММ, логканонические, логтерминальные по Кавамате, чисто логтерминальные особенности, 三, $\sim,\lfloor\cdot\rfloor,\lceil\cdot\rceil,\{\cdot\}, \overline{\mathrm{NE}}(X / Z), a(E, D), \operatorname{discr}(X, D), \operatorname{totaldiscr}(X, D)$; cм. [8], [13], [11]. Всюду ниже, если не оговаривается противное, граница означает $\mathbb{Q}$-границу, т.е. $\mathbb{Q}$-дивизор Вейля $D=\sum d_{i} D_{i}$ такой, что $0 \leqslant d_{i} \leqslant 1$ для всех $i$. Логмногообразие (логпара) $(X / Z \ni o, D)$ - это по определению стягивание $X \rightarrow Z$ вместе с гранищей $D$ на $X$, которое рассматривается локально вблизи слоя над $o \in Z$. Под размерностью логпары $(X / Z \ni o, D)$ мы всегда понимаем размерность тотального пространства $X$.

ОПРЕДЕЛЕНИЕ 1.1 [23]. Пусть $(X / Z, D)$ - логмногообразие. Тогда:

1) численное дополнение - это $\mathbb{R}$-граница $D^{\prime} \geqslant D$ такая, что $K+D^{\prime}$ логканоничен и численно тривиален; 
2) $\mathbb{R}$-дополнение - это $\mathbb{R}$-граница $D^{\prime} \geqslant D$ такая, что $K+D^{\prime}$ логканоничен и $\mathbb{R}$-линейно тривиален;

3) $\mathbb{Q}$-дополнение - это $\mathbb{Q}$-граница $D^{\prime} \geqslant D$ такая, что $K+D^{\prime}$ логканоничен и $\mathbb{Q}$-линейно тривиален;

4) если $D=S+B$, где $S=\lfloor D\rfloor, B=\{D\}$, то $n$-дополнение-это $\mathbb{Q}$-граница $D^{+}$такая, что $K+D^{+}$логканоничен, $n\left(K+D^{+}\right) \sim 0$ и

$$
n D^{+} \geqslant n S+\lfloor(n+1) D\rfloor \text {. }
$$

Заметим, что $\mathbb{R}$-дополнения могут быть рассмотрены как $n$-дополнения для $n=\infty$, так как предел в неравенстве (1.1) при $n \rightarrow \infty$ дает $D^{\prime} \geqslant D$. Все эти определения имеют смысл и в более общей ситуации, когда $D-\mathbb{R}$-субграница (т.е. $\mathbb{R}$-дивизор $D=\sum d_{i} D_{i}$ с $d_{i} \leqslant 1$ для всех $\left.i\right)$.

Очевидно, верны следуюшие импликации:

$$
\exists \mathbb{Q} \text {-дополнение } \Longrightarrow \exists \mathbb{R} \text {-дополнение } \Longrightarrow \exists \text { численное дополнение. }
$$

Простой пример, приведенный ниже, показывает, что $n$-дополнение не обязано быть $\mathbb{Q}$-дополнением (даже численным).

ПримеР 1.2. Пусть $P_{1}, P_{2}, P_{3}$ - различные точки на $\mathbb{P}^{1}$. Положим

$$
D:=P_{1}+\left(\frac{1}{2}+\varepsilon\right) P_{2}+\left(\frac{1}{2}-\varepsilon\right) P_{3}, \quad D^{\prime}:=P_{1}+\frac{1}{2} P_{2}+\frac{1}{2} P_{3},
$$

где $0<\varepsilon \ll 1$. Тогда $K+D^{\prime}$ является 2 -дополнением логдивизора $K+D$. Однако неравенство $D^{\prime} \geqslant D$ неверно, т.е. $K+D^{\prime}$ не является $\mathbb{Q}$-дополнением логдивизора $K+D$.

При дополнительном ограничении на коэффициенты $D$ (например, если коэффициенты $D$ стандартные, см. ниже п. А) неравенство $D^{+} \geqslant D$ имеет место (см. [24, лемма 2.7$]$ или $[20$, п. 4.2$])$, значит, в этом случае $D^{+}-\mathbb{Q}$-дополнение.

Вопрос о существовании дополнений естественно возникает для многообразий типа Фано или Калаби-Яо, т.е. для многообразия с численно эффективным антилогканоническим дивизором, но численная эффективность дивизора $-(K+D)$ не гарантирует существование дополнений [24, пример 1.1].

ПРЕДЛОЖЕНИЕ 1.3 [23, предложение 5.5]. Пусть $(X / Z \ni$ о,D) - логмногообразие. Предположим, что пара $(X, D)$ логканонична и дивизор $-(K+D)$ (полу)обилен над $Z$. Тогда вблизи слоя над о существует $\mathbb{Q}$-дополнение логдивизора $K+D$.

Введем следующие обозначения.

А. Фиксируем подмножество $\Phi \subset[0,1]$. Будем писать $D \in \Phi$, если все коэффициенты дивизора $D$ содержатся в $\Phi$. Например, мы можем рассмотреть $\Phi=$ $\Phi_{\mathbf{s m}}:=\{1-1 / m \mid m \in \mathbb{N} \cup\{\infty\}\}$ (это называется случаем стандартных коәффичиентов). Однако некоторые из наших утверждений и гипотез могут быть сформулированы и для другого выбора $\Phi$ (см., например, (1.3) ниже). 
В. Пусть $(X, D)$ - проективное логмногообразие такое, что:

1) пара $(X, D)$ логканонична;

2) $D \in \Phi$;

3) дивизор $-\left(K_{X}+D\right)$ численно эффективен и объемен;

4) существует некоторое $\mathbb{Q}$-дополнение логдивизора $K_{X}+D$ (это условие выполнено, если дивизор $-\left(K_{X}+D\right)$ полуобилен; например, согласно [8, теорема 3-1-2] оно выполнено, если $K_{X}+D$ логтерминален по Кавамате).

Такие пары мы назьваем логмногообразиями Фано.

В обозначениях п. В определим минимальный индекс дополнений как

$$
\operatorname{compl}(X, D):=\min \left\{m \mid K_{X}+D \quad m \text {-дополняем }\right\}
$$

и рассмотрим множество

$$
\begin{gathered}
N_{d}(\Phi):=\{m \in \mathbb{N} \mid \exists \text { логмногообразие } \Phi \text { ано }(X, D) \text { размерности } d \\
\text { такое, что } D \in \Phi \text { и } \operatorname{compl}(X, D)=m\} .
\end{gathered}
$$

Например, $N_{1}([0,1])=\{1,2,3,4,6\}$ (см. [23]). Рассматривая произведения многообразия $X$ с проективной прямой $\mathbb{P}^{1}$, можно показать, что $N_{d-1}(\Phi) \subset N_{d}(\Phi)$. Положим $N_{0}([0,1])=\{1,2\}$. По индукции определим

$$
\begin{array}{ll}
\Phi_{\mathbf{m}}^{1}:=\Phi_{\mathbf{s} \mathbf{m}}, & N_{1}:=\max N_{1}\left(\Phi_{\mathbf{m}}^{1}\right), \\
\Phi_{\mathbf{m}}^{d}:=\Phi_{\mathbf{s m}} \bigcup\left[1-\frac{1}{N_{d-1}+1}, 1\right], & N_{d}:=\max \left(\bigcup_{k=1}^{d} N_{d}\left(\Phi_{\mathbf{m}}^{k}\right)\right) .
\end{array}
$$

Мы не исключаем случая $N_{d}=\infty$ (и тогда $\Phi_{\mathbf{m}}^{d}:=\Phi_{\mathbf{s m}}$ ), однако мы надеемся, что $N_{d}<\infty$ (см. ниже гипотезу 1.7). Согласно [23, пример 5.2] имеем

$$
N_{1}=6, \quad \Phi_{\mathbf{m}}^{2}=\Phi_{\mathbf{s m}} \cup[6 / 7,1] .
$$

В работе [24] было доказано, что $N_{2}$ конечно. По построению $N_{d} \geqslant N_{d^{\prime}}$ и $\Phi_{\mathbf{m}}^{d} \subset$ $\Phi_{\mathbf{m}}^{d^{\prime}}$, если $d \geqslant d^{\prime}$.

Лемма 1.4 (ср. [24, лемма 2.7]). Если $\alpha \in \Phi_{\mathbf{m}}^{d}$, то для любого $n \leqslant N_{d-1}$ имеем

$$
\lfloor(n+1) \alpha\rfloor \geqslant n \alpha .
$$

ДокАЗАТЕЛЬСТво. Если $\alpha \in \Phi_{\mathbf{s m}}$, то $\alpha=1-1 / m$ для некоторого $m \in \mathbb{N}$. В этом случае мы запишем $n \alpha=q+k / m$, где $q=\lfloor n \alpha\rfloor$ и $k / m=\{n \alpha\}, k \in \mathbb{Z}$, $0 \leqslant k \leqslant m-1$. Тогда

$$
\lfloor(n+1) \alpha\rfloor=\lfloor q+k / m+1-1 / m\rfloor= \begin{cases}q, & \text { если } k=0, \\ q+1 & \text { в противном случае. }\end{cases}
$$

В обоих случаях $\lfloor(n+1) \alpha\rfloor \geqslant q+k / m=n \alpha$. Предположим, что $\alpha \notin \Phi_{\text {sm }}$. Тогда

$$
\alpha>1-\frac{1}{n_{d-1}+1}
$$

и

$$
\lfloor(n+1) \alpha\rfloor \geqslant\left\lfloor n+1-\frac{n+1}{n_{d-1}+1}\right\rfloor \geqslant n \geqslant n \alpha .
$$


СЛЕДСТВИе 1.5. Пусть $(X, D)$ - логпара такая, что $D \in \Phi_{\mathbf{m}}^{d}$, u пусть $D^{+}-n$-дополнение, где $n \leqslant N_{d-1}$. Тогда $D^{+} \geqslant D$.

ЛЕмма 1.6 (ср. [23, лемма 4.2]). Пусть $(X, D)$ - логканоническое логмногообразие, и пусть $S:=\lfloor D\rfloor, B:=\{D\}$. Предположим, что дивизор $K+S$ чисто логтерминален и $D \in \Phi_{\mathbf{m}}^{d}$ для некоторого d (соответственно, $\left.D \in \Phi_{\mathbf{s m}}\right)$. Тогда $\operatorname{Diff}_{S}(B) \in \Phi_{\mathbf{m}}^{d}\left(\right.$ соответственно, $\left.\operatorname{Diff}_{S}(B) \in \Phi_{\mathbf{s m}}\right)$.

ДокаЗАТЕЛЬство. Запишем $B=\sum b_{j} B_{j}, 0<b_{j}<1$. Пусть $\alpha-$ коэффищиент $\operatorname{Diff}_{S}(B)$. Тогда (см. [23, следствие 3.10$\left.]\right)$

$$
\alpha=\frac{m-1}{m}+\sum_{j} \frac{b_{j} n_{j}}{m}
$$

где $m \in \mathbb{N}, n_{j} \in \mathbb{N} \cup\{0\}$. Так как пара $\left(S, \operatorname{Diff}_{S}(B)\right)$ логканонична (см. [13, теорема 17.7]), то $\alpha \leqslant 1$ и можно предположить, что $\alpha<1$. Используя неравенство $b_{j} \geqslant 1 / 2$, легко показать, что в равенстве (1.4) имеет место $\sum n_{j} \leqslant 1$ (см. [23, лемма 4.2]). Если в (1.4) $n_{j}=0$ для всех $j$, то, очевидно, $\alpha \in \Phi_{\mathbf{s m}}$. В противном случае $n_{j_{0}}=1$ для некоторого $j_{0}$ и в (1.4) $n_{j}=0$ для $j \neq j_{0}$. Тогда

$$
\alpha=\left(m-1+b_{j_{0}}\right) / m
$$

Если $b_{j_{0}} \in \Phi_{\mathbf{s m}}$, то $b_{j_{0}}=1-1 / n, n \in \mathbb{N}$, и $\alpha=\frac{m n-1}{m n} \in \Phi_{\mathbf{s m}}$. Если же $b_{j_{0}} \geqslant 1-\frac{1}{N_{d-1}+1}$, то

$$
\alpha \geqslant b_{j_{0}} \geqslant 1-\frac{1}{N_{d-1}+1} .
$$

В обоих случаях $\alpha \in \Phi_{\mathbf{m}}^{d}$.

ГИПОТЕЗА 1.7. В обозначениях $n$. В множество $N_{d}(\Phi)$ конечно.

Доказательство гипотезы 1.7 для размерности два, предложенное в работе [24], сушественным образом использует результаты об ограниченности для логповерхностей дель Пещо [2] (см. также [17]). Для произвольной размерности имеется следующая

ГиПотеЗА 1.8. Фиксируем $\varepsilon>0$. Пусть $(X, D)$ - нормальное проективное логмногообразие такое, что:

1) дивизор $K+D$ является $\mathbb{Q}$-картье;

2) totaldiscr $(X, D)>-1+\varepsilon$;

3) дивизор $-\left(K_{X}+D\right)$ численно эффективен и оббемен.

Тогда многообразие $X$ принадлежит конечному числу алгебраических семейств.

Известно, что эта гипотеза верна при $\operatorname{dim}(X)=2$. Для $\operatorname{dim}(X) \geqslant 3$ имеются только отдельные результаты в этом направлении [3], [4]. Новый подход к доказательству гипотезы 1.8 был предложен в работе $[9, \S 9]$. 
ГИПОТЕЗА 1.9 (индуктивная гипотеза). Пусть пара $(X, D)$ удовлетворяет условиям $n$. В (в частности, $D \in \Phi)$. Предположим, что существует $\mathbb{Q}$-дополнение дивизора $K+D$, которое не логтерминально по Кавамате. Тогда $K+D$ имеет $n$-дополнение для $n \in N_{d-1}(\Phi)$. Более того, это новое дополнение также может быть взято не логтерминальным по Кавамате.

Можно ожидать справедливость гипотезы 1.9 для $\Phi=\Phi_{\mathrm{sm}}$ или $\Phi=\Phi_{\mathbf{m}}^{d}$, где $d=\operatorname{dim}(X)$. Однако в общем случае она неверна [24, пример 2.4], [20, п. 8.1]. В настоящее время эта гипотеза доказана для $\operatorname{dim}(X)=2$ и $\Phi=\Phi_{\mathbf{m}}^{2}[24, \S 2]$ (даже в более сильной форме).

\section{§ 2. Исключительность}

ОПРЕДЕЛЕНИЕ 2.1. МЫ говорим, что стягивание $f: X \rightarrow Z$ имеет локальный mun, если $\operatorname{dim}(Z)>0$. В противном случае (т.е. когда $Z$ - точка) мы говорим, что стягивание $f: X \rightarrow Z$ глобального типа.

Таким образом, стягивание локального типа может быть либо бирациональным, либо расслоенным. В обоих случаях нас интересует структура $f: X \rightarrow Z$ вблизи фиксированного слоя $f^{-1}(o), o \in Z$, и обычно мы считаем, что $X$ - достаточно малая окрестность слоя над $o$.

ОПРЕДЕлЕНИЕ 2.2 (см. $[23, \S 5],[24$, определение 1.5$])$. Пусть $(X / Z \ni o, \Delta)-$ логмногообразие локального типа. Предположим, что $K+\Delta$ имеет по крайней мере одно $\mathbb{Q}$-дополнение вблизи слоя над $о$. Тогда мы назовем логмногообразие $(X / Z \ni o, \Delta)$ исключительнылм, если для любого $\mathbb{Q}$-дополнения ${ }^{1} K+\Delta^{+}$дивизора $K+\Delta$ вблизи слоя над $о$ сушествует не более одного дивизора $E$ поля $K(X)$ такого, что $a\left(E, \Delta^{+}\right)=-1$.

Ясно, что свойство исключительности зависит от выбора базисной точки $o \in Z$. Непосредственно из определения следует

Лемма 2.3. Пусть $(X / Z \ni о, \Delta) u\left(X^{\prime} / Z \ni о, \Delta^{\prime}\right)$ - логмногообразия (локального или глобального типа), и пусть $f: X \rightarrow X^{\prime}$ - стягивание над $Z$. Предположим, что дивизор $K_{X^{\prime}}+\Delta^{\prime}$ является $\mathbb{Q}$-картье $и \Delta$ - крепантный прообраз $\Delta^{\prime}$ (m.е. $\left.f^{*}\left(K_{X^{\prime}}+\Delta^{\prime}\right)=K_{X}+\Delta u f_{*} \Delta=\Delta^{\prime}\right)$. Тогда логмногообразие $(X / Z \ni$ о, $\Delta)$ исключительно, если и только если логмногообразие $\left(X^{\prime} / Z \ni о, \Delta^{\prime}\right)$ является таковим.

ДоКАЗАТЕЛЬСТво следует из [11, предложение 3.10].

ПРЕДЛОЖЕНИЕ 2.4. Пусть $(X / Z \ni о, \Delta)$ - логмногообразие локального типа, и пусть $D, D^{\prime}-\mathbb{Q}$-дополнения такие, что $K+D$ и $K+D^{\prime}$ не логтерминальны по Кавамате. Пусть $S$ и $S^{\prime}$ - простые дивизоры поля $K(X)$ такие, что $a(S, D)=-1$ u $a\left(S^{\prime}, D^{\prime}\right)=-1$. Предположим, что $S \not \approx S^{\prime}$. Тогда существует $\mathbb{Q}$-дополнение $G$ логдивизора $K+\Delta$ такое, что $a(S, G)=a(E, G)=-1$ для некоторого дивизора $E \not \approx S$ поля $K(X)$.

\footnotetext{
${ }^{1}$ Можно считать, что для любого $\mathbb{R}$-дополнения.
} 
ДокАЗАТЕЛЬСТво (ср. [14, предложение 2.7], [7, предложение 2.4]). Заметим, что дивизор $D^{\prime}-D$ является $\mathbb{Q}$-картье и численно тривиален над $Z$. Положим $D(\alpha):=D+\alpha\left(D^{\prime}-D\right)$. Тогда $D(0)=D, D(1)=D^{\prime}$ и $K+D(\alpha)-\mathbb{Q}$-дополнение для всех $0 \leqslant \alpha \leqslant 1$ (по вьпуклости свойства логканоничности см. [23, п. 1.4.1] или [13, предложение 2.17]). Зафиксируем эффективный дивизор Картье $L$ на $Z$ (проходящий через $о$ ) и положим $F:=f^{*} L$. Для $0 \leqslant \alpha \leqslant 1$ определим функцию

$$
\varsigma(\alpha):=\sup \{\beta \mid K+D(\alpha)+\beta F \text { логканоничен }\},
$$

и положим $T(\alpha):=D(\alpha)+\varsigma(\alpha) F$. Зафикисируем некоторое логразрешение $(X, D+$ $\left.D^{\prime}+F\right)$, и пусть $\sum E_{i}$ - объединение исключительного дивизора и собственного прообраза $\operatorname{Supp}\left(D+D^{\prime}+F\right)$. Тогда функция $\varsigma(\alpha)$ может быть вычислена так:

$$
\varsigma(\alpha)=\max _{E_{i}}\left\{\beta \mid a\left(E_{i}, D(\alpha)+\beta F\right) \geqslant-1\right\}
$$

(см., например, [8, п. 0-2-12]). В частности, $\varsigma(\alpha) \in \mathbb{Q}$. Следовательно, логдивизор $K+T(\alpha)$ является $\mathbb{Q}$-дополнением. По рассуждениям, приведенньп вьше, число $\beta=\varsigma(\alpha)$ может быть вычислено из линейных неравенств $a\left(E_{i}, D(\alpha)+\beta F\right) \geqslant-1$, где $E_{i}$ пробегают некоторое конечное число простых дивизоров. Поэтому функция $\varsigma(\alpha)$ кусочно линейна и непрерывна по $\alpha$ и такими же являются коэффициенты дивизора $T(\alpha)$. По построению пара $(X, T(\alpha))$ не логтерминальна по Кавамате для всех $0 \leqslant \alpha \leqslant 1$. Мы утверждаем, что $a(S, T(0))=-1$. В самом деле, $T(0)=$ $D+\varsigma(0) F \geqslant D$. Таким образом, $a(S, T(0)) \leqslant a(S, D)=-1$. Так как дивизор $K+T(0)$ логканоничен, то $a(S, T(0))=-1$. Далее возьмем

$$
\alpha_{0}:=\sup \{\alpha \mid a(S, T(\alpha))=-1\}
$$

По рассуждениям, приведенным выше, $\alpha_{0}$ рационально (и $a\left(S, T\left(\alpha_{0}\right)\right)=-1$ ). Если $\alpha_{0}=1$, то положим $G:=T(1)$ и $E=S^{\prime}$. В противном случае для любых $\alpha>\alpha_{0}$ выполнено неравенство $a(S, T(\alpha))>-1$. Следовательно, существует дивизор $E \not \approx S$ поля $K(X)$ такой, что $a(E, T(\alpha))=-1$. Снова $E$ можно выбрать среди компонент $\sum E_{i}$. Таким образом, $E$ не зависит от $\alpha$, если $0<\alpha-\alpha_{0} \ll 1$. Очевидно, $a\left(E, T\left(\alpha_{0}\right)\right)=-1$, и можно положить $G:=T\left(\alpha_{0}\right)$.

СлЕДСТвИЕ 2.5. Пусть $(X / Z \ni о, \Delta)$ - неисключительное логмногообразие локального типа и $D \geqslant \Delta-\mathbb{Q}$-дополнение такие, что пара $(X, D)$ не является логтерминальной по Кавамате. Пусть $S$-дивизор поля $K(X)$ такой, что $a(S, D)=-1$. Тогда имеется $\mathbb{Q}$-дополнение $G \geqslant \Delta$ такое, что $a(S, G)=a(E, G)=-1$ для некоторого дивизора $E \not \approx S$ поля $K(X)$.

ДокАзАТЕльСтво. Так как пара $(X / Z \ni o, \Delta)$ неисключительна, то имеется $\mathbb{Q}$-дополнение $D^{\prime} \geqslant \Delta$ такое, что $a\left(S^{\prime}, D^{\prime}\right)=-1$ для некоторого $S^{\prime} \not \approx S$. Далее применяем предложение 2.4 .

СлЕДСТвИЕ 2.6. Пусть $(X / Z \ni о, \Delta)$ - исключительное логмногообразие локального типа. Тогда существует однозначно определенный дивизор $S$ поля $K(X)$ такой, что для любого $\mathbb{Q}$-дополнения $D$ имеем $a(E, D)>-1$, если $E \not \approx S$ в $K(X)$.

Назовем дивизор $S$, определенный в следствии 2.6 , центральным дивизором исключительной логпары $(X / Z \ni o, \Delta)$. 
СЛЕДСТВИЕ 2.7. Пусть $(X / Z \ni$ о, $\Delta)$ - исключительное логмногообразие локального типа и $S$ - чентральный дивизор. Тогда иентр $S$ на $X$ содержится в слое над о.

ДокАЗАТЕЛЬСТво. Пусть $K+D$ - $\mathbb{Q}$-дополнение такое, что $a(S, D)=-1$, и пусть $H$ - обшее гиперплоское сечение многообразия $Z$, проходящее через $o$. Если $f^{*} H$ не содержит центр $S$, то mult $_{S} f^{*} H=0$ и $a(S, D)=a\left(S, D+c f^{*} H\right)=-1$ для всех $c$. Возьмем $c$, для которого $K+D+c f^{*} H$ максимально логканоничен. Тогда, как и в доказательстве предложения $2.4, a\left(E, D+c f^{*} H\right)=-1$ для некоторого $E \not ॅ S$. Противоречие.

ПримеР 2.8. Рассмотрим логканоническую особенность $X \ni$ о (т.е. $X=Z$ и $\Delta=0)$. Тогда эта особенность исключительна, если и только если для любой границы $B$ на $X$ такой, что пара $(X, B)$ логканонична, существует не более одного дивизора $E$ поля $K(X)$ такого, что $a(E, B)=-1$. Например, двумерная логтерминальная особенность исключительна, если и только если она имеет тип $\mathbb{E}_{6}, \mathbb{E}_{7}$ или $\mathbb{E}_{8}$ (см. [23, пример 5.2.3], [14]).

В глобальном случае определение 2.2 выглядит несколько иначе.

ОПРЕДЕЛЕНИЕ 2.9. Пусть $(X, \Delta)$ - логмногообразие глобального типа. Предположим, что дивизор $K+\Delta$ имеет хотя бы одно $n$-дополнение. Тогда говорят, что пара $(X, \Delta)$ исключительна, если любое $\mathbb{Q}$-дополнение $K+\Delta^{+}$дивизора $K+\Delta$ логтерминально по Кавамате (т.е. $a\left(E, \Delta^{+}\right)>-1$ для любого дивизора $E$ поля $K(X))$.

ПримеР 2.10. і) Пусть $X=\mathbb{P}^{1}, Z$ - точка и

$$
\Delta=\sum_{i=1}^{r}\left(1-1 / m_{i}\right) P_{i}, \quad m_{i} \in \mathbb{N},
$$

где $P_{1}, \ldots, P_{r}$ - различные точки. Дивизор $-(K+\Delta)$ численно эффективен, если и только если $\sum_{i=1}^{r}\left(1-1 / m_{i}\right) \leqslant 2$. В этом случае набор $\left(m_{1}, \ldots, m_{r}\right)$ дает нам исключительную пару, если и только если он (с точностью до перестановок) один из следуюших:

$$
\begin{array}{llllll}
E_{6}: & (2,3,3), & E_{7}: & (2,3,4), & E_{8}: & (2,3,5), \\
\widetilde{E}_{6}: & (3,3,3), & \widetilde{E}_{7}: & (2,4,4), & \widetilde{E}_{8}: & (2,3,6), \\
\widetilde{D}_{4}: & (2,2,2,2) . & & & &
\end{array}
$$

ii) Пусть $X=\mathbb{P}^{d}, Z$ - точка и

$$
\Delta=\sum_{i=1}^{d+2}\left(1-1 / m_{i}\right) \Delta_{i}, \quad m_{i} \in \mathbb{N}
$$

где $\Delta_{1}, \ldots, \Delta_{d+2}$ - гиперплоскости в $\mathbb{P}^{d}$. Логдивизор $-(K+\Delta)$ численно эффективен, если и только если $\sum 1 / m_{i} \leqslant 1$. Если логпара $(X, \Delta)$ исключительна, то 
дивизор $-\left(K+\Delta_{j}+\sum_{i \neq j}\left(1-1 / m_{i}\right) \Delta_{i}\right)$ не является численно эффективным для всех $j$. Следовательно, $\sum_{i \neq j} 1 / m_{i}>1$. Теперь нетрудно показать существование констант Const $(d)$ таких, что $m_{j} \leqslant$ Const $(d)$ для всех $j$ (cp. [11, пример 8.16]). Поэтому имеется только конечное число возможностей для исключительных наборов $\left(m_{1}, \ldots, m_{d+2}\right)$.

Эти примеры и многие другие факты (см. [24], [14], [7], [19], [6], [21]) показывают, что в общей ситуации можно ожидать выполнения следующих принщипов:

1) неисключительные пары имеют хорошие свойства линейной системы $|-m(K+D)|$ для некоторого малого $m$;

2) исключительные пары могут быть классифицированы.

\section{§ 3. Стягивания Фано}

В этом параграфе мы докажем теорему 3.1. Двумерная версия этого результата была доказана вторым автором в [23]. Затем это было обобщено в [24], [19].

ТЕОРема 3.1 (локальный случай). Пусть $\Phi:=\Phi_{\mathbf{m}}^{d}\left(\right.$ или $\left.\Phi:=\Phi_{\mathbf{s m}}\right)$, и пусть $(X / Z \ni о, D)$ - d-мерное логмногообразие локального типа такое, что:

1) $D \in \Phi$;

2) пара $(X, D)$ логтерминальна по Кавамате;

3) дивизор - $(K+D)$ численно әффективен и обгемен над $Z$.

Пусть $f: X \rightarrow Z$ - структурный морфизм. Предположим, что логПММ верна в размерности $d$. Тогда существует не логтерминальное по Кавамате п-дополнение дивизора $K+D$ вблизи $f^{-1}($ o) для некоторого челого $n \in N_{d-1}(\Phi)$. Более того, если пара $(X / Z \ni$ о, D) неисключительна и гипотеза 1.9 выполнена в размерностях $d^{\prime} \leqslant d-1$ для $\Phi=\Phi_{\mathbf{m}}^{d}$ (соответственно, $\left.\Phi=\Phi_{\mathbf{s m}}\right)$, то дивизор $K+D$ n-дополняем вблизи слоя $f^{-1}($ о) для $n \in N_{d-2}(\Phi)$. Это дополнение также может быть выбрано неисключительHbl.M.

В неисключительном случае мы ожидаем более точных результатов; тогда особенности дополнения должны зависеть от топологической структуры существенного исключительного дивизора (см. $[24, \S 7])$.

ПРИмеР 3.2. Пусть ( $Z \ni o)$ - двумерная дювалевская особенность (рациональная двойная точка), $D=0$ и $f$ - тождественное отображение. Имеется не логтерминальное по Кавамате $n$-дополнение дивизора $K_{Z}$ для некоторого $n \in$ $N_{1}\left(\Phi_{\mathbf{s m}}\right)=\{1,2,3,4,6\}$ (см. [23, пример 5.2.3]). Особенность неисключительна, если она имеет тип $A_{n}$ или $D_{n}$. В этих случаях имеется не логтерминальное по Кавамате $n$-дополнение для $n \in N_{0}\left(\Phi_{\mathbf{s m}}\right)=\{1,2\}$.

Ключевая идея доказательства теоремы 3.1 проста: мы находим некоторое специальное раздутие $X$ с неприводимым исключительным дивизором $S$ (предложение 3.6) и затем применяем индуктивные свойства дополнений (предложение 6.2) для того, чтобы свести проблему к многообразию $S$ меньшей размерности (но уже, возможно, проективному). 
Лемма 3.3. Пусть $(X / Z, D)$ - логмногообразие такое, что пара $(X, D)$ логтерминальна по Кавамате и дивизор $-\left(K_{X}+D\right)$ численно әффективен и оббемен над $Z$. Тогда существует әффективный $\mathbb{Q}$-дивизор $D^{\mho}$ такой, что пара $\left(X, D+D^{\mho}\right)$ снова логтерминальна по Кавамате, а дивизор $-\left(K_{X}+D+\right.$ $\left.D^{\mho}\right)$ обилен над $Z$.

ДокАЗАТЕЛЬСТво следует из леммы Кодаиры (см., например, [8, лемма 0-3-3]).

СлЕДСТВИЕ 3.4. В обозначениях и предположениях леммы 3.3 конус Мори $\overline{\mathrm{NE}}(X / Z)$ полиэдрален и порождается стягиваемыми экстремальным.ми рачиональнымми кривыми.

ОПРЕДЕЛЕНИЕ 3.5 . Пусть $(X, \Delta)$ - логмногообразие и $g: Y \rightarrow X-$ раздутие такое, что исключительное множество $g$ содержит в точности один неприводимьй дивизор $S$. Предположим, что пара $\left(Y, \Delta_{Y}+S\right)$ чисто логтерминальна и дивизор $-\left(K_{Y}+\Delta_{Y}+S\right) g$-обилен. Тогда $g:(Y \supset S) \rightarrow X$ назьвается иисто логтерминальным раздутием пары $(X, \Delta)$.

В отличие от логтерминальных модификаций [25, теорема 3.1] чисто логтерминальные раздутия не являются логкрепантными.

Пусть $(X \ni o, D)$ - исключительная особенность. Тогда по следствию 2.6 имеется не более одного чисто логтерминального раздутия (см. [18, предложение 6$]$ ).

ПРЕДЛОЖЕНИЕ 3.6 (см. [18], [20, п. 3.1], ср. [26]). Пусть $\left(X, \Delta+\Delta^{0}\right)-$ логмногообразие такое, что многообразие $X \mathbb{Q}$-факториально, $\Delta \geqslant 0, \Delta^{0} \geqslant 0$, дивизор $K+\Delta+\Delta^{0}$ логканоничен, но не чисто логтерминален, а $K+\Delta$ логтерминален по Кавамате. (Мы не утверждаем, что $\Delta u \Delta^{0}$ не имеют общих компонент.) Предположим, что верна логПММ в размерности $\operatorname{dim}(X)$. Тогда существует иисто логтерминальное раздутие $g:(Y \supset S) \rightarrow X$ парь $(X, \Delta)$ такое, что:

1) дивизор $K_{Y}+\Delta_{Y}+S+\Delta_{Y}^{0}=g^{*}\left(K+\Delta+\Delta^{0}\right)$ логканоничен;

2) дивизор $K_{Y}+\Delta_{Y}+S+(1-\varepsilon) \Delta_{Y}^{0}$ чисто логтерминален и антиобилен над $X$ для любых $\varepsilon>0$;

3) многообразие $Y \mathbb{Q}$-факториально и $\rho(Y / X)=1$.

Раздутия такого рода мы называем индуктивными раздутиями пары $(X, \Delta+$ $\left.\Delta^{0}\right)$. Важно отметить, что это определение зависит от $\Delta$ и $\Delta^{0}$, а не только от $\Delta+\Delta^{0}$. Такие раздутия часто применяются в теории дополнений. В локальном случае можно сконструировать гранищу $\Delta^{0}$ такую, как в предложении 3.6 , добавляя полный прообраз некоторого $\mathbb{Q}$-дивизора на $Z$. В глобальном случае проблема построения $\Delta^{0}$ значительно сложнее.

ДокАЗАТЕЛЬСТво. Рассмотрим сначала логтерминальную модификацию ${ }^{2}$ $h: V \rightarrow X$ логпары $\left(X, \Delta+\Delta^{0}\right)$ (см. [23], [13, теорема 17.10]). Запишем

$$
h^{*}\left(K+\Delta+\Delta^{0}\right)=K_{V}+\Delta_{V}+\Delta_{V}^{0}+E,
$$

\footnotetext{
${ }^{2}$ Иногда логтерминальная модификация называется также логтерминальньлм разрешением.
} 
где $\Delta_{V}$ и $\Delta_{V}^{0}-$ собственные прообразы $\Delta$ и $\Delta^{0}$ соответственно, а $E$ - исключительный дивизор. Можно выбрать модификацию $h$ таким образом, что дивизор $E$ приведен и $E \neq 0$ (см. [13, теорема 17.10], [25, теорема 3.1]). Мы утверждаем, что дивизор $K_{V}+\Delta_{V}+E$ не может быть численно эффективен над $X$. В самом деле, запишем

$$
h^{*}(K+\Delta)=K_{V}+\Delta_{V}+\sum \alpha_{i} E_{i}
$$

где $\alpha_{i}<1$ для всех $i$. Это дает нам

$$
h^{*} \Delta^{0}=\Delta_{V}^{0}+\sum\left(1-\alpha_{i}\right) E_{i}
$$

Таким образом,

$$
K_{V}+\Delta_{V}+E \equiv-\Delta_{V}^{0} \equiv \sum\left(1-\alpha_{i}\right) E_{i} \text { над } X
$$

где дивизор $\sum\left(1-\alpha_{i}\right) E_{i}$ эффективен, исключителен и не равен нулю. Этот дивизор не может быть $h$-численно эффективен (см., например, [23, п. 1.1]). Далее применим $\left(K_{V}+\Delta_{V}+E\right)$-ПММ над $X$. При последнем шаге мы получаем бирациональное стягивание $g: Y \rightarrow X$, которое удовлетворяет условиям 1)-3) предложения 3.6.

Теорему 3.1 будем доказывать индукцией по $d$. Таким образом, предположим, что утверждение теоремы 3.1 выполнено при $\operatorname{dim}(X)<d$. Заменим многообразие $X$ на его $\mathbb{Q}$-ракториализацию (см. [13, теорема 6.11.1]). Это сохраняет все наши предположения. После этого рассмотрим дивизор $D^{\mho}$ такой, как в лемме 3.3 , и положим $D^{\nabla}:=D^{\mho}+c f^{*} H$, где $H$-эффективный дивизор Картье на $Z$, проходяший через $о$, и $c$ - логканонический порог:

$$
c=c\left(X, D+D^{\mho}, f^{*} H\right)
$$

(максимальное число такое, что $K+D+D^{\mho}+c f^{*} H$ логканоничен).

Тогда верно следующее

УТВЕРЖДЕНИЕ 3.7. Дивизор $K+D+D^{\nabla}$ антиобилен над $Z$, логканоничен и не логтерминален по Кавамате.

Заметим, что дивизоры $D$ и $D^{\nabla}$ могут иметь обшие компоненты. Далее мы выделяем два случая:

(a) пара $\left(X, D+D^{\nabla}\right)$ чисто логтерминальна (и $\left.\left\lfloor D+D^{\nabla}\right\rfloor \neq 0\right)$;

(b) пара $\left(X, D+D^{\nabla}\right)$ не является чисто логтерминальной.

В случае (В) мы рассмотрим индуктивное раздутие $g: \widehat{X} \rightarrow X$ пары $(X, D+$ $D^{\nabla}$ ). Пусть $S$ - (неприводимый) исключительный дивизор. Согласно [23, лемма 5.4] (или [13, лемма 19.2]) достаточно доказать существование искомого дополнения на $\widehat{X}$. Запишем

$$
\begin{aligned}
g^{*}\left(K+D+D^{\nabla}\right) & =K_{\widehat{X}}+\Delta+S+\widehat{D}^{\nabla}, \\
g^{*}(K+D) & =K_{\widehat{X}}+\Delta+a S,
\end{aligned}
$$


где $\widehat{D}^{\nabla}$ и $\Delta-$ собственные прообразы $D^{\nabla}$ и $D$ соответственно, а $a<1$. Заметим, что $\Delta+a S$ необязательно является гранищей.

В случае (а) мы положим $\widehat{X}=X, g=\mathrm{id}, S=\left\lfloor D+D^{\nabla}\right\rfloor$. В этом случае многообразие $S$ неприводимо по лемме о связности [13, теорема 17.4 ] и потому, что многообразие $S$ нормально [13, следствие 17.5]. Определим дивизор $\Delta$ из равенства $D=\Delta+a S$, где $0 \leqslant a<1$ и $S$ не является компонентой $\Delta$. Положим

$$
\widehat{D}^{\nabla}:=D+D^{\nabla}-S-\Delta .
$$

В обоих случаях (a) и (b) мы имеем согласно утверждению 3.7 и равенству (3.1) следуюшее утверждение (см. [11, предложение 3.10$])$.

УТВЕРЖДЕНИЕ 3.8. Пара $\left(\widehat{X}, \Delta+S+\widehat{D}^{\nabla}\right)$ логканонична, не логтерминальна по Кавамате, пара $(\widehat{X}, \Delta+a S)$ логтерминальна по Кавамате, и оба дивизора $-\left(K_{\widehat{X}}+\Delta+S+\widehat{D}^{\nabla}\right) u-\left(K_{\widehat{X}}+\Delta+a S\right)$ численно әффективны и обтемны над $Z$.

ЛЕмма 3.9. Существуют $\delta_{0}>0$ и граница $M$ на $\widehat{X}$ такие, что:

1) имеет место неравенство

$$
\Delta+a S \leqslant M \leqslant \Delta+S+\left(1-\delta_{0}\right) \widehat{D}^{\nabla}
$$

2) пара $(\widehat{X}, M)$ логтерминальна по Кавамате;

3) дивизор $-(K+M)$ численно әффективен и облемен над $Z$.

В частности, конус Мори $\overline{\mathrm{NE}}(\widehat{X} / Z)$ полиэдрален.

ДокАЗАТЕльСтво. Согласно утверждению 3.7 дивизор $K+D+\left(1-\delta_{0}\right) D^{\nabla}$ логтерминален по Кавамате и антиобилен над $Z$ для достаточно малых положительных $\delta_{0}$. Возьмем $M$ как крепантный прообраз:

$$
\begin{aligned}
K_{\widehat{X}}+M & =g^{*}\left(K+D+\left(1-\delta_{0}\right) D^{\nabla}\right) \\
& =g^{*}(K+D)+\left(1-\delta_{0}\right)\left(g^{*}\left(K+D+D^{\nabla}\right)-g^{*}(K+D)\right) \\
& =K_{\widehat{X}}+\Delta+a S+\left(1-\delta_{0}\right)\left(\left(K_{\widehat{X}}+\Delta+S+\widehat{D}^{\nabla}\right)-\left(K_{\widehat{X}}+\Delta+a S\right)\right) .
\end{aligned}
$$

Его можно записать еще так:

$$
\begin{aligned}
M & =\Delta+a S+\left(1-\delta_{0}\right)\left(S+\widehat{D}^{\nabla}-a S\right) \\
& =\Delta+\left(1-\delta_{0}(1)\right) S+\left(1-\delta_{0}\right) \widehat{D}^{\nabla} .
\end{aligned}
$$

Из равенства (3.3) получаем, что пара $(\widehat{X}, M)$ логтерминальна по Кавамате [11, предложение 3.10], а дивизор - $(K+M)$ численно эффективен и объемен над $Z$. Неравенство (3.2) заведомо выполнено, если $a \leqslant 1-\delta_{0}(1)$, т.е. для $0<\delta_{0} \ll 1$.

Далее определим вспомогательный дивизор $\widehat{D}^{\lambda}$.

А. Возьмем $0<\lambda \ll \delta_{0}$ и положим

$$
\widehat{D}^{\lambda}:=(1-\lambda) \widehat{D}^{\nabla}
$$


Утверждаем, что логдивизор $K_{\widehat{X}}+\Delta+S+\widehat{D}^{\lambda}$ чисто логтерминален и антиобилен над $Z$.

В самом деле, в случае (b), так как $\rho(\widehat{X} / X)=1$, кривые в слоях морфизма $g$ порождают экстремальный луч $R$. Тогда

$$
R \cdot\left(K_{\widehat{X}}+\Delta+S+\widehat{D}^{\nabla}\right)=0
$$

(и дивизор $K_{\widehat{X}}+\Delta+S+\widehat{D}^{\nabla}$ строго отрицателен на всех экстремальных лучах, не равных $R$; см. утверждение 3.7 и равенство (3.1)). Далее, учитывая (3.1), имеем $\widehat{D}^{\nabla} \equiv-(1-a) S$ над $X$, и дивизор $\widehat{D}^{\nabla}$ положителен на $R$. Таким образом, логдивизор $K_{\widehat{X}}+\Delta+S+\widehat{D}^{\lambda}$ строго отрицателен на всех экстремальных лучах конуса $\overline{\mathrm{NE}}(\widehat{X} / Z)$ для достаточно малых положительных $\lambda$. Тогда по критерию Клеймана он антиобилен. Наконец, пара $\left(\widehat{X}, \Delta+S+\widehat{D}^{\lambda}\right)$ чисто логтерминальна потому, что $\widehat{D}^{\lambda} \leqslant \widehat{D}^{\nabla}$. В случае (а) наше утверждение, очевидно, следует из утверждения 3.7 .

Заметим, что $M \leqslant \Delta+S+\widehat{D}^{\lambda}$ согласно неравенству (3.2).

Теперь мы можем построить границу $B$.

В. Зафиксируем некоторое множество $F_{1}, \ldots, F_{r}$ главных дивизоров на $\widehat{X}$. Для $n \gg 0$ рассмотрим обший элемент

$$
F \in\left|-n\left(K_{\widehat{X}}+\Delta+S+\widehat{D}^{\lambda}\right)-\sum F_{i}\right|
$$

и положим

$$
B:=\widehat{D}^{\lambda}+\frac{1}{n}\left(F+\sum F_{i}\right)
$$

Можно выбрать $F_{1}, \ldots, F_{r}$ и $n$ таким образом, что:

1) пара $(\widehat{X}, \Delta+S+B)$ чисто логтерминальна;

2) компоненты дивизора $B$ порождают $N^{1}(\widehat{X} / Z)$.

Наша конструкция задает численную эквивалентность:

$$
K+\Delta+S+B \equiv 0 \text { над } Z \text {. }
$$

Возьмем $\varepsilon>0$ таким образом, чтобы дивизор $K+\Delta+S+(1+\varepsilon) B$ был чисто логтерминален (см. [13, предложение 2.17]) и

$$
M \leqslant \Delta+S+(1-\varepsilon) B
$$

(т.е. $1-\delta_{0} \leqslant(1-\varepsilon)(1-\lambda)$; см. доказательство леммы 3.9). Применим $(K+\Delta+$ $S+(1+\varepsilon) B)-П М М$ над $Z$ :

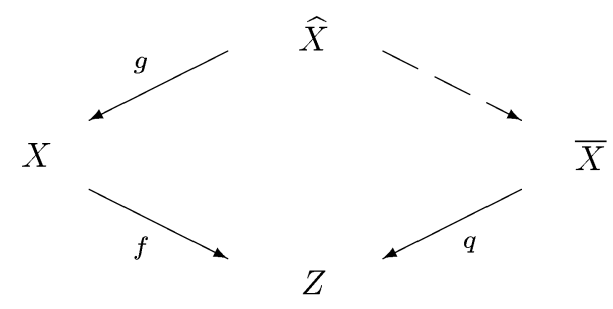


Ниже мы используем черту, например $\bar{\Delta}$, для обозначения собственного прообраза на $\bar{X}$ дивизора, определенного на $\widehat{X}$. Для каждого экстремального луча $R$ вьполнены неравенства $R \cdot B<0$ и $R \cdot(K+\Delta+S)>0$. Поэтому любое стягивание является либо мальм, либо дивизориальным и стягивает компоненту $B$. В частности, любое дивизориальное стягивание не стягивает $S$. На последнем шаге ПММ получим, что дивизор $(K+\Delta+S+(1+\varepsilon) B)$ численно эффективен над $Z$ (не исключаем случая $\bar{X}=Z)$. Так как $K+\Delta+S+B \equiv 0$, то дивизор $-(K+\Delta+S)$ также численно эффективен над $Z$.

ЛЕмма 3.10. Можно применить $(K+\Delta+S+(1+\varepsilon) B)$-ПММ таким образом, что на каждом шаге возможно найти гранииу $M \leqslant \Delta+S+(1-\varepsilon) B$ такую, что дивизор $K+M$ логтерминален по Кавамате и дивизор $-(K+M)$ численно эффективен и оббемен над $Z$.

ДокАЗАТЕЛЬСтво. По лемме 3.9 такая гранища сушествует уже на первом шаге. Если дивизор $K+\Delta+S+(1+\varepsilon) B \equiv \varepsilon B$ не численно эффективен над $Z$, то $-(K+\Delta+S+(1-\varepsilon) B) \equiv \varepsilon B$ также не численно эффективен над $Z$. Положим

$$
t_{0}:=\sup \{t \mid-(K+M+t(\Delta+S+(1-\varepsilon) B-M)) \text { численно эффективен }\} .
$$

По лемме 3.4 этот супремум является максимумом и достигается на некотором экстремальном луче. Следовательно, $t_{0}$ рационально и $0<t_{0}<1$. Рассмотрим границу

$$
M^{0}:=M+t_{0}(\Delta+S+(1-\varepsilon) B-M) .
$$

Тогда дивизор $-\left(K+M^{0}\right)$ численно эффективен над $Z$ и $M^{0} \leqslant \Delta+S+(1-\varepsilon) B$. Утверждаем, что дивизор $-\left(K+M^{0}\right)$ также объемен над $Z$. Предположим противное. По теореме о стабильной свободе дивизор $-\left(K+M^{0}\right)$ полуобилен над $Z$ и определяет стягивание $\varphi: \widehat{X} \rightarrow W$ на многообразие меньшей размерности. Пусть $C$ - общая кривая в слое. Тогда

$$
C \cdot\left(K+M^{0}\right)=C \cdot(K+\Delta+S+B)=0 .
$$

Таким образом, $C \cdot\left(\Delta+S+B-M^{0}\right)=0$. Так как кривая $C$ численно эффективна, то $\varepsilon C \cdot B \leqslant C \cdot\left(\Delta+S+B-M^{0}\right)=0$ и $C \cdot B=0$. По свойству 2$)$ п. В имеем $C \equiv 0$. Противоречие.

Далее, конус $\overline{\mathrm{NE}}(\widehat{X} / Z)$ полиэдрален. Поэтому имеется экстремальный луч $R$ такой, что $R \cdot\left(K+M^{0}\right)=0$ и

$$
\varepsilon R \cdot B=-r \cdot(K+\Delta+S+(1-\varepsilon) B)<0 .
$$

Следовательно, $R \cdot(K+\Delta+S+(1+\varepsilon) B)<0$. Пусть $h: \widehat{X} \rightarrow Y$ - стягивание $R$. Положим $M_{Y}^{0}:=h_{*} M^{0}$. Тогда

$$
K+M^{0}=h^{*}\left(K_{Y}+M_{Y}^{0}\right)
$$

Поэтому дивизор $K_{Y}+M_{Y}^{0}$ является $\mathbb{Q}$-картье. Пара $\left(Y, M_{Y}^{0}\right)$ логтерминальна по Кавамате, а дивизор $-\left(K_{Y}+M_{Y}^{0}\right)$ численно эффективен и объемен над $Z$. Если 
стягивание $g$ дивизориально, то можно продолжить процесс, заменяя $\widehat{X}$ на $Y$ и $M$ на $M=M_{Y}^{0}$. Предположим, что $g$ - малое стягивание, и пусть

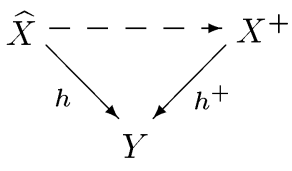

- его перестройка (флип). Положим $M:=h^{+-1}\left(M_{Y}^{0}\right)$. Тогда снова дивизор $-\left(K_{X^{+}}+M^{+}\right)=-h^{+*}\left(K_{Y}+M_{Y}^{0}\right)$ численно эффективен и объемен над $Z$. Таким образом, мы можем продолжить процесс, заменяя $X$ на $X^{+}$.

С. Применим ПММ такую, как в лемме 3.10. На последнем шаге получим многообразие $\bar{X}$ такое, что:

1) пара $(\bar{X}, \bar{\Delta}+\bar{S})$ чисто логтерминальна;

2) дивизор $-(K+\bar{\Delta}+\bar{S})$ численно эффективен над $Z$.

Лемма 3.11. Дивизор $-(K+\bar{\Delta}+\bar{S})$ полуобилен над $Z$. Более того, если $-(K+\bar{\Delta}+\bar{S})$ не обилен, то он определяет бирачиональное стягивание над $Z$ с исключительным множеством, содержсашимся в $\operatorname{Supp}(\bar{B})$. В частности, дивизор

$$
-\left(K_{\bar{S}}+\operatorname{Diff}_{\bar{S}}(\bar{\Delta})\right)=-\left.(K+\bar{\Delta}+\bar{S})\right|_{\bar{S}}
$$

обтемен (и численно эффективен) над $q(\bar{S})$.

ДокАЗАТЕЛЬСТво. По лемме 3.10 и по теореме о стабильной свободе дивизор $-(K+\bar{\Delta}+\bar{S})$ полуобилен. Таким образом, для некоторого $n \in \mathbb{N}$ линейная система $|-n(K+\bar{\Delta}+\bar{S})|$ определяет стягивание $\bar{X} \rightarrow W$. Для любой кривой $C$ в слое имеем $C \cdot \bar{B}=0$. Поскольку компоненты $\bar{B}$ порождают $N^{1}(\bar{X} / Z)$ (см. свойство 2) п. В), то $C \cdot \bar{B}_{i}<0$ для некоторой компоненты $\bar{B}_{i}$ дивизора $\bar{B}$. Следовательно, $C \subset \operatorname{Supp}(\bar{B})$.

Заметим, что $q: \bar{S} \rightarrow q(\bar{S})$ - также стягивание.

ЛЕмма 3.12. Имеет место равенство $q_{*} \mathscr{O}_{\bar{S}}=\mathscr{O}_{q(\bar{S})}$, и многообразие $q(\bar{S})=f(g(S))$ нормально.

ДокАЗАТЕЛьСтво. См. доказательство леммы 3.6 из [23].

Согласно лемме 1.6 имеем $\operatorname{Diff}_{\bar{S}}(\bar{\Delta}) \in \Phi$ (напомним, что мы положили $\Phi=\Phi_{\mathbf{m}}^{d}$ или $\left.\Phi=\Phi_{\mathrm{sm}}\right)$.

ЛЕМма 3.13. Предположим, что вблизи слоя $q^{-1}($ о) существует $n$-дополнение $K_{\bar{S}}+\operatorname{Diff}_{\bar{S}}(\bar{\Delta})^{+}$дивизора $K_{\bar{S}}+\operatorname{Diff}_{\bar{S}}(\bar{\Delta})$. Тогда вблизи $q^{-1}($ о) существует также $n$-дополнение $K+D^{+}$дивизора $K+D$. Более того, если пара $\left(\bar{S}, \operatorname{Diff}_{\bar{S}}(\bar{\Delta})^{+}\right)$не логтерминальна по Кавамате, то пара $\left(X, D^{+}\right)$не является исключительной. 
ДоКАЗАТЕЛЬСТВо. Согласно предложению 6.2 (см. ниже) любое $n$-дополнение дивизора $K_{\bar{S}}+\operatorname{Diff}_{\bar{S}}(\bar{\Delta})$ может быть продолжено до $n$-дополнения дивизора $K+$ $\bar{\Delta}+\bar{S}$, а по предложению 6.1 мы можем брать прообразы дополнения дивизора $K+\Delta+S$ при дивизориальных стягиваниях, так как все эти стягивания $(K+\Delta+S)$-положительны. Наконец, заметим, что перестройки сохраняют дополнения, т. е. собственньй прообраз $n$-дополнения при перестройке снова является $n$-дополнением. В самом деле, неравенство (1.1), очевидно, сохраняется при любом бирациональном отображении, являющимся изоморфизмом для коразмерности один. Свойство логканоничности и линейной эквивалентности из определения 1.1 также сохраняются [13, предложение 2.28].

Лемма 3.14. $E c л u \operatorname{dim}(q(\bar{S}))>0, m o$ :

i) пара $(X / Z \ni о, D)$ неисключительна;

ii) имеется неисключительное $n$-дополнение дивизора $K+D, n \in N_{d-2}(\Phi)$.

ДокАЗАТЕЛЬСтво. Утверждение і) следует из следствия 2.7. Заметим, что пара $\left(\bar{S} / q(\bar{S}) \ni o, \operatorname{Diff}_{\bar{S}}(\bar{\Delta})\right)$ удовлетворяет условиям нашей теоремы (см. лемму 1.6). По предположению индукции можно считать, что имеется не логтерминальное по Кавамате $n$-дополнение $K_{\bar{S}}+\operatorname{Diff}_{\bar{S}}(\bar{\Delta})$ для $n \in N_{d-2}(\Phi)$. Остальное получается из леммы 3.13 .

Возвратимся к доказательству теоремы 3.1 .

D. Допустим, что пара $(X / Z \ni o, D)$ неисключительна (т.е. существует неисключительное дополнение $K+D+\Upsilon)$ и $q(\bar{S})=o$. Достаточно показать, что существует неисключительное $n$-дополнение дивизора $K+D$ с $n \in N_{d-2}(\Phi)$. Coгласно лемме 3.14 можно предположить, что $q(\bar{S})=o$, т.е. многообразие $\bar{S}$ проективно. По следствию 2.5 дивизор $\Upsilon$ можно выбрать так, что $a(S, D+\Upsilon)=-1$ (и $a(E, D+\Upsilon)=-1$ для некоторого $E \not \approx S)$. Пусть $\widehat{\Upsilon}$ и $\bar{\Upsilon}-$ собственные прообразы $\Upsilon$ на $\widehat{X}$ и $\bar{X}$ соответственно. Тогда

$$
g^{*}(K+D+\Upsilon)=K_{\widehat{X}}+\Delta+S+\widehat{\Upsilon} .
$$

Более того,

$$
a(E, \Delta+S+\widehat{\Upsilon})=a(E, \bar{\Delta}+\bar{S}+\bar{\Upsilon})=-1
$$

(так как $K_{\widehat{X}}+\Delta+S+\widehat{\Upsilon} \equiv 0$ ). Таким образом, дивизор $K_{\bar{X}}+\bar{\Delta}+\bar{S}+\bar{\Upsilon}$ не является чисто логтерминальным (вблизи слоя $\left.q^{-1}(o)\right)$.

ЛЕмма 3.15. В предположениях $n$. D дивизор $K_{\bar{S}}+\operatorname{Diff}_{\bar{S}}(\bar{\Delta}+\bar{\Upsilon})$ не является логтерминальным по Кавамате.

ДокАЗАТЕЛЬСТво. По формуле присоединения [13, теорема 17.6] достаточно доказать, что дивизор $K+\bar{\Delta}+\bar{S}+\bar{\Upsilon}$ не является чисто логтерминальным вблизи $\bar{S}$. Учитывая рассуждения, приведенные вьше, мы видим, что последнее является следствием леммы 3.17 .

Согласно лемме 3.15 и гипотезе 1.9 получаем, что имеется не логтерминальное по Кавамате $n$-дополнение $K_{\bar{S}}+\operatorname{Diff}_{\bar{S}}(\bar{\Delta})$, где $n \in N_{d-2}(\Phi)$. По лемме 3.13 это доказывает теорему 3.1 .

Следуюший пример поясняет доказательство теоремы 3.1. 
ПримеР 3.16. Как и в примере 3.2, пусть $(Z \ni o)$ - двумерная дювалевская особенность (рациональная двойная точка), пусть $D=0$, и пусть $f$ - тождественное отображение. В этом случае $g: \widehat{X} \rightarrow X$-взвешенное раздутие (с подходяшими весами) и $\widehat{X} \rightarrow \bar{X}$ - тождественное отображение. Следовательно, $S \simeq \mathbb{P}^{1}$. Запишем

$$
\operatorname{Diff}_{S}(0)=\sum_{i=1}^{r}\left(1-1 / m_{i}\right) P_{i}
$$

где $P_{1}, \ldots, P_{r}$ - различные точки. Имеем следующее соответствие между типами $(Z \ni o)$ и наборами $\left(m_{1}, \ldots, m_{r}\right)$ (см. примеры 2.8 и 2.10$)$ :

\begin{tabular}{c||c|c|c|c|c}
$(Z \ni o)$ & $A_{n}$ & $D_{n}$ & $E_{6}$ & $E_{7}$ & $E_{8}$ \\
\hline$\left(m_{1}, \ldots, m_{r}\right)$ & $r \leqslant 2$ & $(2,2, m)$ & $(2,3,3)$ & $(2,3,4)$ & $(2,3,5)$
\end{tabular}

Таким образом, особенность $(Z \ni o)$ исключительна, если и только если она имеет тип $E_{6}, E_{7}$ или $E_{8}$.

ЛЕмма 3.17 (см. [19], ср. [23, теорема 6.9], [5, предложение 2.1]). Пусть $(X / Z \ni$ о,D) - логмногообразие, и пусть $f: X \rightarrow Z$ - структурный морфизм. Предположим, что:

1) дивизор $K+D$ логканоничен и не чисто логтерминален вблизи $f^{-1}(o)$;

2) $K+D \equiv 0$ над $Z$;

3) имеется неприводимая компонента $S \subset\lfloor D\rfloor$ такая, что $f(S) \neq Z$.

Предположим также выполнимость логПММ в размерности $\operatorname{dim}(X)$. Тогда пара $(X, D)$ не является чисто логтерминальной вблизи $S \cap f^{-1}($ о).

СлЕДСТВИЕ 3.18. В обозначениях теоремь 3.1 следующие утверждения әквивалентны:

i) $(X / Z \ni о, D)$ - исключительная пара (локального типа);

ii) $q(\bar{S})=$ о $и\left(\bar{S}, \operatorname{Diff}_{\bar{S}}(\bar{D})\right)$ - исключительная пара (глобального типа).

ДокАЗАТЕЛЬСТвО. Импликация і) $\Longrightarrow$ іi) следует из леммы 3.14 и леммы 3.13 . Обратная импликация - из леммы 3.15 .

Положим

$$
\operatorname{compl}^{\prime}(X, D):=\min \left\{\begin{array}{l|l}
m \mid \begin{array}{l}
\text { существует не логтерминальное по Кавамате } \\
m \text {-дополнение дивизора } K+D
\end{array}
\end{array}\right\} .
$$

СЛЕДСТВИЕ 3.19. В обозначениях и условиях теоремы 3.1 предположим дополнительно, что пара $(X / Z \ni$ о,D) исключительна. Тогда

$$
\operatorname{compl}^{\prime}(X, D)=\operatorname{compl}\left(\bar{S}, \operatorname{Diff}_{\bar{S}}(\bar{D})\right) .
$$


ДокаЗАтЕльство. Неравенство $\operatorname{compl}^{\prime}(X, D) \leqslant \operatorname{compl}\left(\bar{S}, \operatorname{Diff}_{\bar{S}}(\bar{D})\right)$ следует из леммы 3.13. Таким образом, мы будем доказывать обратное неравенство. Пусть $K+D^{+}$- не логтерминальное по Кавамате $n$-дополнение дивизора $K+D$. Тогда $D^{+} \geqslant D$. По следствию 2.6 имеем $a\left(S, D^{+}\right)=-1$. Рассмотрим крепантньй прообраз

$$
g^{*}\left(K+D^{+}\right)=K_{\widehat{X}}+\Delta+S+\Upsilon,
$$

и пусть $\bar{\Upsilon}$ - собственный прообраз дивизора $\Upsilon$ на $\bar{X}$. Тогда $K_{\bar{S}}+\operatorname{Diff}_{\bar{S}}(\bar{\Delta}+\bar{\Upsilon})-$ $n$-дополнение дивизора $K_{\bar{S}}+\operatorname{Diff}_{\bar{S}}(\bar{\Delta})$.

Заметим, что для неисключительных стягиваний мы имеем только неравенство $\operatorname{compl}^{\prime}(X, D) \leqslant \operatorname{compl}\left(\bar{S}, \operatorname{Diff}_{\bar{S}}(\bar{D})\right)$.

ПримеР 3.20. Пусть $(X \ni o)$ - терминальная $c E_{8}$-особенность, заданная уравнением

$$
x_{1}^{2}+x_{2}^{3}+x_{3}^{5}+x_{4}^{r}=0,
$$

где н. о. д. $(r, 30)=1$, и пусть $g:(\widehat{X}, S) \rightarrow X-$ взвешенное раздутие с весами $(15 r, 10 r, 6 r, 30)$. Тогда $S=\mathbb{P}^{2}$ и

$$
\operatorname{Diff}_{S}(0)=\frac{1}{2} L_{1}+\frac{2}{3} L_{2}+\frac{4}{5} L_{3}+\frac{r-1}{r} L_{4}
$$

где $L_{1}, \ldots, L_{4}$ - прямые на $\mathbb{P}^{2}$ в общем положении. Тогда $\operatorname{compl}^{\prime}(X, 0)=1$ (так как $(X \ni o)-c D V$-особенность). С другой стороны, $\operatorname{compl}\left(\bar{S}, \operatorname{Diff}_{\bar{S}}(0)\right)=6$.

\section{§ 4. Исключительные стягивания Фано}

В этом параграфе изучены исключительные стягивания Фано, удовлетворяющие условиям теоремы 3.1 .

ПРЕДЛОЖЕНИЕ 4.1. Сохраним обозначения и предположения теоремы 3.1. Предположим также, что гипотеза 1.7 верна для размерностей не более $d-1$. Пусть пара $(X / Z \ni$ э,D) исключительна. Тогда

$$
a(E, D) \geqslant-1+\delta_{d} \quad \text { для всех } E \not \approx S,
$$

где $\delta_{d}>0$ - константа, зависящая только от $d$.

ДоказАТЕЛЬСтво. Пусть $K+D^{+}-$не логтерминальное по Кавамате $n$-дополнение, где $n \in N_{d-1}(\Phi)$. Тогда $D^{+} \geqslant D$ (см. следствие 1.5$)$. По определению исключительных стягиваний $a\left(S, D^{+}\right)=-1$ и $a\left(E, D^{+}\right)>-1$ для всех $E \not \approx S$. Следовательно, $a\left(E, D^{+}\right) \geqslant-1+1 / n$ (так как число $n a\left(E, D^{+}\right)$целое). Так как $D^{+} \geqslant D$, то $a(E, D) \geqslant a\left(E, D^{+}\right)$. Таким образом, подходит

$$
\delta_{d}:=1 / \max \left(N_{d-1}\left(\Phi_{\mathbf{m}}^{d-1}\right)\right) .
$$

Предполагая, что $D \in \Phi_{\mathbf{s m}}$, получаем 
СлЕДСТВИЕ 4.2. Сохраним обозначения и предположения предложения 4.1. Пусть $D_{i}-$ компонента $D$, и пусть $d_{i}=1-1 / m_{i}-$ ее коэффициент. Если $D_{i} \not \approx S$, то $m_{i} \leqslant 1 / \delta(d)$, и поэтому имеется лишь конечное число значений $d_{i}$.

СлЕДСТВИЕ 4.3 (ср. [10]). Предположим, ито верны логПММ для размерностей не более $d$ и гипотеза 1.9 для размерностей не более $d-1$. Пусть $(X \ni$ $о)-d$-мерная логтерминальная по Кавамате особенность, и пусть $F=\sum F_{i}-$ эффективный приведенный дивизор Вейля $\mathbb{Q}$-картве на $X$, проходящий через о. Тогда выполнено одно из следующих условий:

1) $c_{0}(X, F)=1$,

2) $c_{0}(X, F) \leqslant 1-1 / N_{d-1}$,

где $c_{0}(X, F)$ - логканонический порог $(X, F)$ (cм. [23], а также [11]) и $N_{d-1}-$ константа, определенная в (1.3).

Отметим, что это следствие является нетривиальным, если только гипотеза 1.7 верна для размерностей не более $d-1$.

ДокАЗАТЕЛЬСТво. Положим $c:=c_{0}(X, F)$ и предположим, что $1-1 / N_{d-1}<$ $c<1$. Из теоремы 3.1 следует существование $n$-дополнения $K+B$ дивизора $K+c F$, где $n \leqslant N_{d-1}$. Пусть $c_{i}^{+}$- коэффициент компоненты $F_{i}$ в $B$. В силу неравенства (1.1) имеем $c_{i}^{+} \geqslant 1$. Следовательно, $F \leqslant B$ и дивизор $K+F$ логканоничен. Противоречие.

В случае, когда

$$
1-1 /\left(N_{d-2}+1\right) \leqslant c=c_{0}(X, F)<1,
$$

пара $(X, c F)$ исключительна. Мы ожидаем, что для любой размерности имеется только конечное число значений для $c \in\left[1-1 /\left(N_{d-2}+1,1\right)\right]$. Например, этот метод дает (см., например, [20, следствие 6.0.9]), что для размерности $d=2$ множество всех значений $c_{0}(X, F)$ на отрезке $[2 / 3,1]$ совпадает с множеством $\{2 / 3,7 / 10,3 / 4,5 / 6,1\}$.

Теорема 4.4. Фиксируем $\varepsilon>0$. Пусть $(X / Z \ni o, D)-d$-мерное логмногообразие локального типа такое, что:

1) $D \in \Phi_{\mathbf{s m}}$ (m.е. $d=\sum\left(1-1 / m_{i}\right) D_{i}$, где $m_{i} \in \mathbb{N} \cup\{\infty\}$ и $D_{i}-$ главные дивизоры);

2) имеет место неравенство

$$
\text { totaldiscr }(X, D)>-1+\varepsilon
$$

3) дивизор $-(K+D)$ численно эффективен и обғемен над $Z$;

4) пара $(X / Z \ni о, D)$ исключительна.

Далее, пусть $\varphi: X^{\prime} \rightarrow X$ - конечное накрытие такое, что:

5) многообразие $X^{\prime}$ нормально и неприводимо;

6) морфизм $\varphi$ әтален для коразмерности один вне $\operatorname{Supp}(D)$;

7) индекс ветвления морфизма $\varphi$ в общей точке компонент дивизора $\varphi^{-1}\left(D_{i}\right)$ делит $m_{i}$.

Предположим также, что логПММ верна для размерностей не более $d u$ для $\Phi_{\mathrm{sm}}$ верны гипотезы 1.8, 1.7 и 1.9 для размерности $d-1$. Тогда степень $\varphi$ ограничена константой Const $(d, \varepsilon)$. 
ДоКАЗАТЕЛЬСТВо. Воспользуемся обозначениями доказательства теоремы 3.1. Рассматривая расслоенное произведение многообразия $X$ с его $\mathbb{Q}$-факториализацией, можно свести ситуацию к случаю, когда многообразие $X \mathbb{Q}$-факториально. Заметим также, что слой $\varphi^{-1} \circ f^{-1}(o)$ связен (так как $X$ рассматривается как росток вблизи слоя $f^{-1}\left(\right.$ ) и многообразие $X^{\prime}$ неприводимо). Рассмотрим коммутативную диаграмму

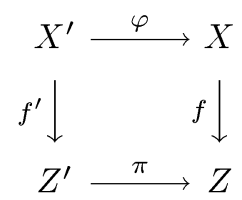

где $X^{\prime} \stackrel{f^{\prime}}{\rightarrow} Z^{\prime} \stackrel{\pi}{\rightarrow} Z$ - разложение Штейна. Тогда $f^{\prime}: X^{\prime} \rightarrow Z^{\prime}$ - стягивание и $\pi: Z^{\prime} \rightarrow Z$ - конечный морфизм. Определим дивизоры $D^{\prime}$ и $D^{\nabla \prime}$ следуюшим образом:

$$
\begin{aligned}
K_{X^{\prime}}+D^{\prime} & =\varphi^{*}(K+D), \\
K_{X^{\prime}}+D^{\prime}+D^{\nabla \prime} & =\varphi^{*}\left(K+D+D^{\nabla}\right)
\end{aligned}
$$

(см. $[23, \S 2])$. Это означает, что, например, коэффициент компоненты $D_{i, j}^{\prime}$ дивизора $\varphi^{-1}\left(D_{i}\right)$ в $D^{\prime}$ имеет следуюший вид:

$$
d_{i, j}^{\prime}=1-r_{i, j}\left(1-\left(1-1 / m_{i}\right)\right),
$$

где $r_{i, j}$-индекс ветвления в обшей точке $D_{i, j}^{\prime}$. Тогда по условию 7$)$ нашей теоремы имеем $D^{\prime} \in \Phi_{\mathbf{s m}}$ (и $D^{\nabla \prime} \geqslant 0$ ). Очевидно, $K_{X^{\prime}}+D^{\prime}+D^{\nabla \prime}$ обилен над $Z^{\prime}$.

Сначала рассмотрим случай (а) из $§ 3$ (т.е. случай, когда дивизор $K+D+D^{\nabla}$ чисто логтерминален, $S:=\left\lfloor D+D^{\nabla}\right\rfloor \neq 0, \widehat{X}=X$ и $g$ - тождественное отображение). Положим $S^{\prime}:=\left\lfloor D^{\prime}+D^{\nabla \prime}\right\rfloor=\varphi^{-1}(S)$. Согласно лемме 3.14 многообразие $S$ компактно и $S \subset f^{-1}(o)$. Применяя [23, 2 ] (или [13, предложение 20.3]), получаем, что пара $\left(X^{\prime}, D^{\prime}+D^{\nabla \prime}\right)$ чисто логтерминальна. По лемме о связности $[13$, теорема 17.4] и по формуле присоединения [13, теорема 17.6] многообразие $S^{\prime}$ связно, неприводимо и нормально. Определим дивизор $\Delta^{\prime}$ из равенства $D^{\prime}=\Delta^{\prime}+a^{\prime} S^{\prime}$, где $0 \leqslant a^{\prime}<1$. Пусть $\bar{X}^{\prime}-$ нормализация многообразия $\bar{X}$ в поле функций многообразия $X^{\prime}$. Имеется коммутативная диаграмма

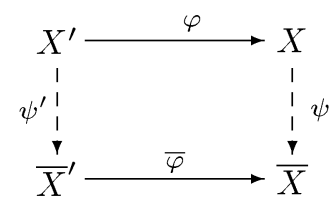

где $\bar{\varphi}: \bar{X}^{\prime} \rightarrow \bar{X}$ - конечный морфизм, а $\psi: X \rightarrow \bar{X}$ и $\psi^{\prime}: X^{\prime} \rightarrow \bar{X}^{\prime}-$ бирациональные отображения такие, что $\psi^{-1}$ и $\psi^{\prime-1}$ не стягивают дивизоров. Следовательно, накрытие $\bar{\varphi}$ имеет дивизор ветвления только над $\operatorname{Supp}\left(\psi_{*}(D)\right) \subset \bar{S} \cup \operatorname{Supp}(\bar{\Delta})$. Индекс ветвления $\bar{\varphi}$ в обшей точке компоненты над $\psi_{*}\left(D_{i}\right)$ равен индексу ветвления $\varphi$ в общей точке соответствующей компоненты над $D_{i}$. Применяя $\psi_{*}$ и $\psi_{*}^{\prime}$ к соотношениям (4.2), получим

$$
\begin{aligned}
K_{\bar{X}^{\prime}}+\bar{D}^{\prime} & =\bar{\varphi}^{*}\left(K_{\bar{X}}+\bar{D}\right), \\
K_{\bar{X}^{\prime}}+\bar{D}^{\prime}+\bar{D}^{\nabla \prime} & =\bar{\varphi}^{*}\left(K_{\bar{X}}+\bar{D}+\bar{D}^{\nabla}\right),
\end{aligned}
$$


где $\bar{D}^{\prime}:=\psi_{*}^{\prime} D^{\prime}$ и $\bar{D}^{\nabla \prime}:=\psi_{*}^{\prime} D^{\nabla \prime}$. Напомним, что $\bar{S}=\left\lfloor\bar{D}+\bar{D}^{\nabla}\right\rfloor-$ простой дивизор. Далее, из первого равенства (4.3) получаем

$$
K_{\bar{X}^{\prime}}+\bar{\Delta}^{\prime}+\bar{S}^{\prime}=\bar{\varphi}^{*}\left(K_{\bar{X}}+\bar{\Delta}+\bar{S}\right)
$$

где $\bar{\Delta}^{\prime}:=\psi_{*}^{\prime} \Delta^{\prime}$ и $\bar{S}^{\prime}:=\psi_{*}^{\prime} S^{\prime}$. Согласно $[23, \S 2]$ и утверждению 1$)$ п. С (см. также $\left[13\right.$, предложение 20.3]) дивизор $K_{\bar{X}^{\prime}}+\bar{\Delta}^{\prime}+\bar{S}^{\prime}$ чисто логтерминален. Более того, утверждение 2$)$ п. С и лемма 3.11 дают нам, что дивизор $-\left(K_{\bar{X}^{\prime}}+\bar{\Delta}^{\prime}+\bar{S}\right)^{\prime}$ численно эффективен и объемен над $Z^{\prime}$. Достаточно доказать ограниченность степени морфизма-ограничения $\bar{\phi}=\left.\bar{\varphi}\right|_{\bar{S}^{\prime}}: \bar{S}^{\prime} \rightarrow \bar{S}$. В самом деле, $\operatorname{deg} \varphi=(\operatorname{deg} \bar{\phi}) r$, где $r-$ индекс ветвления над $S$. Согласно неравенству (4.1) и условию 7) теоремы 4.4 индекс $r$ ограничен. Далее мы рассмотрим логпары $\left(\bar{S}, \operatorname{Diff}_{\bar{S}}(\bar{\Delta})\right)$ и $\left(\bar{S}^{\prime}, \operatorname{Diff}_{\bar{S}^{\prime}}\left(\bar{\Delta}^{\prime}\right)\right)$.

Ограничивая соотношение (4.4) на $\bar{S}$, получаем

$$
K_{\bar{S}}+\operatorname{Diff}_{\bar{S}}(\bar{\Delta})=\bar{\phi}^{*}\left(K_{\bar{S}^{\prime}}+\operatorname{Diff}_{\bar{S}^{\prime}}\left(\bar{\Delta}^{\prime}\right)\right)
$$

В частности,

$$
\left(K_{\bar{S}}+\operatorname{Diff}_{\bar{S}}(\bar{\Delta})\right)^{d-1}=(\operatorname{deg} \bar{\phi})\left(K_{\bar{S}^{\prime}}+\operatorname{Diff}_{\bar{S}^{\prime}}\left(\bar{\Delta}^{\prime}\right)\right)^{d-1} .
$$

Обе части этого равенства положительны по лемме 3.11 .

Ограничим степень морфизма $\bar{\phi}$ (в случае (а)).

Е. Из доказательства теоремы 3.1 видно, что имеется $n$-дополнение $K_{\bar{X}}+\bar{\Delta}+$ $\bar{S}+\bar{\Upsilon}$ логдивизора $K_{\bar{X}}+\bar{\Delta}+\bar{S}$, где $n \leqslant \max N_{d-1}\left(\Phi_{\mathbf{s m}}\right)<\infty$. Зададим дивизор $\bar{\Upsilon}^{\prime}$ равенством

$$
K_{\bar{X}^{\prime}}+\bar{\Delta}^{\prime}+\bar{S}^{\prime}+\bar{\Upsilon}^{\prime}=\bar{\varphi}^{*}\left(K_{\bar{X}}+\bar{\Delta}+\bar{S}+\bar{\Upsilon}\right)
$$

и положим $\Theta:=\operatorname{Diff}_{\bar{S}}(\bar{\Delta}+\bar{\Upsilon}), \Theta^{\prime}:=\operatorname{Diff}_{\bar{S}^{\prime}}\left(\bar{\Delta}^{\prime}+\bar{\Upsilon}^{\prime}\right)$. Тогда $K_{\bar{S}}+\Theta$ и $K_{\bar{S}^{\prime}}+\Theta^{\prime}$ являются $n$-дополнениями. Так как дивизор $K_{\bar{S}}+\Theta$ логтерминален по Кавамате (см. следствие 3.18 ), то

$$
\text { totaldiscr }\left(\bar{S}, \operatorname{Diff}_{\bar{S}}(\bar{\Delta})\right) \geqslant-1+\frac{1}{n} \geqslant-1+\beta, \quad \beta=\frac{1}{\max N_{d-1}\left(\Phi_{\mathrm{sm}}\right)} .
$$

Аналогично,

$$
\text { totaldiscr }\left(\bar{S}^{\prime}, \operatorname{Diff}_{\bar{S}^{\prime}}\left(\bar{\Delta}^{\prime}\right)\right) \geqslant-1+\beta .
$$

Согласно гипотезе 1.8 пары $\left(\bar{S}, \operatorname{Supp}\left(\operatorname{Diff}_{\bar{S}}(\bar{\Delta})\right)\right)$ и $\left(\bar{S}^{\prime}, \operatorname{Supp}\left(\operatorname{Diff}_{\bar{S}^{\prime}}\left(\bar{\Delta}^{\prime}\right)\right)\right)$ принадлежат конечному числу алгебраических семейств. Учитывая то, что $\operatorname{Diff}_{\bar{S}}(\bar{\Delta})$, $\operatorname{Diff}_{\bar{S}^{\prime}}\left(\bar{\Delta}^{\prime}\right) \in \Phi_{\mathbf{s m}}($ см. лемму 1.6), и неравенства

$$
\operatorname{Diff}_{\bar{S}}(\bar{\Delta}) \leqslant \Theta, \quad \operatorname{Diff}_{\bar{S}^{\prime}}\left(\bar{\Delta}^{\prime}\right) \leqslant \Theta^{\prime}
$$

получим, что пары $\left(\bar{S}, \operatorname{Diff}_{\bar{S}}(\bar{\Delta})\right)$ и $\left(\bar{S}^{\prime}, \operatorname{Diff}_{\bar{S}^{\prime}}\left(\bar{\Delta}^{\prime}\right)\right)$ также принадлежат конечному числу алгебраических семейств. Это дает нам ограниченность степени $\operatorname{deg} \bar{\phi}$. 
Далее мы рассмотрим случай (b) из $\S 3$. Пусть $\widehat{X}^{\prime}$ - нормализация доминантной компоненты произведения $\widehat{X} \times_{X} X^{\prime}$ и $S^{\prime}-$ собственный прообраз $S$ на $\widehat{X}^{\prime}$. Утверждается, что морфизм $g^{\prime}:\left(\widehat{X}^{\prime} \supset S^{\prime}\right) \rightarrow X^{\prime}$ является чисто логтерминальным раздутием пары $\left(X^{\prime}, D^{\prime}\right)$. Рассмотрим замену базы

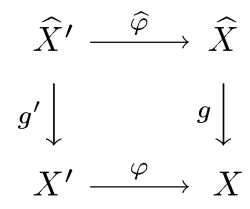

Ясно, что морфизм $\widehat{\varphi}: \widehat{X}^{\prime} \rightarrow \widehat{X}$ конечен и его дивизор ветвления может содержаться только в $S \cup \operatorname{Supp}(D)$. Тогда $S^{\prime}-$ исключительный дивизор раздутия $g^{\prime}: \widehat{X}^{\prime} \rightarrow X^{\prime}$. Действительно,

$$
K_{\widehat{X}^{\prime}}+\Delta^{\prime}+S^{\prime}=\widehat{\varphi}^{*}\left(K_{\widehat{X}}+\Delta+S\right)
$$

где $\Delta^{\prime}$ - граница. Этот дивизор чисто логтерминален [23, следствие 2.2], [13, предложение 20.3] и антиобилен над $X^{\prime}$. По формуле присоединения [13, теорема 17.6] многообразие $S^{\prime}$ нормально. С другой стороны, $S^{\prime}$ связно вблизи слоя над $o^{\prime} \in Z^{\prime}$. В самом деле, $-\left(K_{\widehat{X}^{\prime}}+\widehat{D}^{\prime}+\widehat{D}^{\nabla \prime}\right)$ численно эффективен и объемен над $Z^{\prime}$ по (4.5) и утверждению 3.8 . Так как $S^{\prime} \subset\left\lfloor\widehat{D}^{\prime}+\widehat{D}^{\nabla \prime}\right\rfloor$, то многообразие $S^{\prime}$ связно по лемме о связности [13, теорема 17.4]. Это доказывает наше утверждение.

Далее, как в случае (а) из $\S 3$, мы рассмотрим коммутативную диаграмму

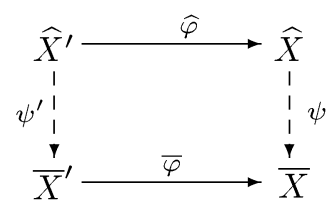

Аналогично случаю (а) пары $\left(\bar{S}, \operatorname{Diff}_{\bar{S}}(\bar{\Delta})\right)$ и $\left(\bar{S}^{\prime}, \operatorname{Diff}_{\bar{S}^{\prime}}\left(\bar{\Delta}^{\prime}\right)\right)$ ограничены. Следовательно, мы можем предположить, что степень $\operatorname{deg} \bar{\phi}$ ограничена, где $\bar{\phi}=\left.\bar{\varphi}\right|_{\bar{S}^{\prime}}$ : $\bar{S}^{\prime} \rightarrow \bar{S}$. Остается показать, что индекс ветвления $r$ морфизма $\bar{\varphi}$ в общей точке $S^{\prime}$ ограничен. Ясно, что $r$ совпадает с индексом ветвления $\widehat{\varphi}$ в общей точке $\widehat{S}^{\prime}$. Аналогично равенству (3.1) запишем

$$
g^{*}\left(K_{X^{\prime}}+D^{\prime}\right)=K_{\widehat{X}^{\prime}}+\Delta^{\prime}+a^{\prime} S^{\prime}
$$

Тогда

$$
1-a^{\prime}=r(1-a) \geqslant r(1+\operatorname{discr}(X, D))>r \varepsilon
$$

(см. $[23, \S 2]$ или $\left[13\right.$, доказательство 20.3]). Утверждается, что пары $\left(S^{\prime}, \operatorname{Diff}_{S^{\prime}}\left(\Delta^{\prime}\right)\right)$ принадлежат конечному числу алгебраических семейств. Заметим, что мы не можем применить гипотезу 1.8 непосредственно потому, что дивизор $-\left(K_{S^{\prime}}+\right.$ $\left.\operatorname{Diff}_{S^{\prime}}\left(\Delta^{\prime}\right)\right)$ необязательно численно эффективен. Как и в случае (а) из $\S 3$, возь- 
мем $n$-дополнение $K_{\widehat{X}}+\Delta+S+\widehat{\Upsilon}$, где $n \leqslant \max N_{d-1}\left(\Phi_{\mathbf{s m}}\right)$. Аналогично (4.2) определим дивизоры $\widehat{\Upsilon}^{\prime}$ и $\widehat{D}^{\lambda \prime}$ (см. п. А):

$$
\begin{aligned}
K_{\widehat{X}^{\prime}}+\Delta^{\prime}+S^{\prime}+\widehat{\Upsilon}^{\prime} & =\widehat{\varphi}^{*}\left(K_{\widehat{X}}+\Delta+S+\widehat{\Upsilon}\right) \\
K_{\widehat{X}^{\prime}}+\Delta^{\prime}+S^{\prime}+\widehat{D}^{\lambda \prime} & =\widehat{\varphi}^{*}\left(K_{\widehat{X}}+\Delta+S+\widehat{D}^{\lambda}\right) .
\end{aligned}
$$

Тогда

$$
K_{S^{\prime}}+\operatorname{Diff}_{S^{\prime}}\left(\Delta^{\prime}+\widehat{\Upsilon}^{\prime}\right) \equiv 0
$$

и по п. А дивизор $K_{S^{\prime}}+\operatorname{Diff}_{S^{\prime}}\left(\Delta^{\prime}+\widehat{D}^{\lambda \prime}\right)$ антиобилен. Следовательно, дивизор $-\left(K_{S^{\prime}}+\operatorname{Diff}_{S^{\prime}}\left(\Delta^{\prime}+\alpha \widehat{D}^{\lambda \prime}+(1-\alpha) \widehat{\Upsilon}^{\prime}\right)\right)$ обилен для любого $\alpha>0$. Заметим, что

$$
\text { totaldiscr }\left(S^{\prime}, \operatorname{Diff}_{S^{\prime}}\left(\Delta^{\prime}+\widehat{\Upsilon}^{\prime}\right)\right) \geqslant-1+1 / n
$$

Таким образом, можно применить гипотезу $1.8 \mathrm{k}$ паре $\left(S^{\prime}, \operatorname{Diff}_{S^{\prime}}\left(\Delta^{\prime}+\alpha \widehat{D}^{\lambda \prime}+\right.\right.$ $\left.\left.(1-\alpha) \widehat{\Upsilon}^{\prime}\right)\right)$ для малого положительного $\alpha$. Получим, что многообразие $S^{\prime}$ ограничено. Далее, как и в п. Е, мы видим, что пара $\left(S^{\prime}, \operatorname{Diff}_{S^{\prime}}\left(\Delta^{\prime}\right)\right)$ также ограничена. Возьмем достаточно обшую кривую $\ell$ в общем слое $\left.g^{\prime}\right|_{S^{\prime}}: S^{\prime} \rightarrow g^{\prime}(S)^{\prime}$. Из равенства (4.7) имеем

$$
-\left(K_{S^{\prime}}+\operatorname{Diff}_{S^{\prime}}\left(\Delta^{\prime}\right)\right) \cdot \ell=-\left(1-a^{\prime}\right) S^{\prime} \cdot \ell
$$

Ясно, что индекс пересечения $-\left(K_{S^{\prime}}+\operatorname{Diff}_{S^{\prime}}\left(\Delta^{\prime}\right)\right) \cdot \ell$ зависит только от пары $\left(S^{\prime}, \operatorname{Diff}_{S^{\prime}}\left(\Delta^{\prime}\right)\right)$, но не от $\widehat{X}^{\prime}$. Таким образом, мы предполагаем, что число $-\left(K_{S^{\prime}}+\right.$ $\left.\operatorname{Diff}_{S^{\prime}}\left(\Delta^{\prime}\right)\right) \cdot \ell$ фиксировано. Напомним, что коэффициенты $\operatorname{Diff}_{S^{\prime}}\left(\Delta^{\prime}\right)$ стандартные (см. [23, предложение 3.9], [13, предложение 16.6]), таким образом, можно записать

$$
\operatorname{Diff}_{S^{\prime}}\left(\Delta^{\prime}\right)=\sum_{i=1}^{r}\left(1-1 / m_{i}\right) \Xi_{i}^{\prime}, \quad m_{i} \in \mathbb{N}, \quad r \geqslant 0
$$

Положим $m^{\prime}:=$ н. о. к. $\left(m_{1}, \ldots, m_{r}\right)$. Согласно [23, предложение 3.9$]$ дивизоры $m^{\prime} S^{\prime}$ и $m^{\prime}\left(K_{S^{\prime}}+\operatorname{Diff}_{S^{\prime}}\left(\Delta^{\prime}\right)\right)$ являются дивизорами Картье вдоль $\ell$. Поэтому (4.9) может быть переписано как $N=\left(1-a^{\prime}\right) k$, где $N=-m^{\prime} \ell \cdot\left(K_{S^{\prime}}+\operatorname{Diff}_{S^{\prime}}\left(\Delta^{\prime}\right)\right)-$ фиксированное натуральное число и $k=-m^{\prime}(\ell \cdot S)^{\prime}$ - также натуральное. Значит,

$$
N=\left(1-a^{\prime}\right) k>k r \varepsilon \geqslant r \varepsilon
$$

по неравенству (4.8). Это показывает, что индекс ветвления $r$ ограничен: $r<N / \varepsilon$, что и доказывает теорему.

Далее мы укажем несколько следствий теоремы 3.1 и теоремы 4.4. В основном нас занимает трехмерный случай (когда известно, что все требуемые гипотезы истинны; см. [24] и [2]). Напомним, что в этом случае неисключительные стягивания такие, как в теореме 3.1 , имеют 1-, 2-, 3-, 4- или 6-дополнение. Положим в теореме $4.4 X=Z$ и $D=0$. Получим 
СлеДСТвИЕ 4.5. Пусть $(Z \ni$ э $D)$ - трехмерная исключительная логтерминальная по Кавамате особенность такая, что

$$
\text { totaldiscr }(Z, D)>-1+\varepsilon
$$

u $D \in \Phi_{\mathbf{s m}} \cdot$ Тогда:

1) порядок алгебраической фундаментальной группь $\pi_{1}^{\operatorname{alg}}(Z \backslash \operatorname{Sing}(Z))$ ограничен константой Const $(\varepsilon)$;

2) индекс $K_{Z}+D$ ограничен константой Const $(\varepsilon)$;

3) для любого исключительного дивизора $E$ над $Z$ имеет место одно из двух: или $а(E)>0$, или $a(E) \in \mathfrak{M}(\varepsilon)$, где $\mathfrak{M}(\varepsilon) \subset(-1,0]-$ подмножество, зависящее только от $\varepsilon$.

Заметим, что без предположения исключительности порядок $\pi_{1}^{\operatorname{alg}}(Z \backslash \operatorname{Sing}(Z))$ не обязательно ограничен (тем не менее, это множество конечно [22, теорема 3.6$]$ ). Утверждение 1) следствия 4.5 также выполнено для топологической фундаментальной группы $\pi_{1}$ при предположении, что она конечна. М. Рид сообщил авторам, что конечность $\pi_{1}(Z \backslash \operatorname{Sing}(Z))$ для трехмерной логтерминальной особенности была доказаны Н. Шепард-Барроном (не опубликовано).

СлЕДСТВИЕ 4.6 [19]. Фиксируем $\varepsilon>0$. Пусть $(X / Z \ni о, D)-$ трехмерное логмногообразие локального типа такое, что $K+D$ - дивизор $\mathbb{Q}$-картье и $-(K+D)$ является $f$-численно эффективным и $f$-оббемным. Предположим, что стягивание $f$ исключительно и

$$
\text { totaldiscr }(X)>-1+\varepsilon
$$

1) Eсли $\operatorname{dim}(Z) \geqslant 2$, то порядок группь $\pi_{1}^{\mathrm{alg}}(Z \backslash \operatorname{Sing}(Z))$ ограничен константой Const $(\varepsilon)$.

2) Если $\operatorname{dim}(Z)=1$, то кратность центрального слоя $f^{-1}($ о) ограничена константой Const $(\varepsilon)$.

СлеДСТВИЕ 4.7 [24]. Фиксируем $\varepsilon>0$. Пусть $(X / Z \ni o, D)-$ трехмерная исключительная логпара такая, что структурный морфизм $f: X \rightarrow Z \ni о-$ малое стягивание (т.е. $f$ стягивает только конечное число кривых),

$$
\text { totaldiscr }(X, D)>-1+\varepsilon
$$

$D \in \Phi_{\mathbf{m}}^{3}$ и дивизор $-(K+D)$ численно әффективен и оббемен над $Z$. Тогда:

1) относительнье числа Пикара $\rho(X / Z)$ и $\rho^{\text {an }}(X / Z)$ ограничень констанmoü Const $(\varepsilon)$;

2) число компонент центрального слоя $f^{-1}($ о) ограничено константой Const $^{\prime}(\varepsilon)$. 
ДоКАЗАТЕЛЬСтво. Сохраним обозначения, принятые в доказательстве теоремы 3.1. Возьмем некоторое $n$-дополнение $K_{\widehat{X}}+\Delta+S+\Upsilon$, где $n \leqslant N_{2}$. Применим $\left(K_{\widehat{X}}+\Delta+\Upsilon\right)$-ПММ. Для каждого экстремального луча $R$ имеем $R \cdot S>0$. Следовательно, $S$ не стягивается. В конце мы получим модель $p: \widetilde{X} \rightarrow Z$ с $p$-численно эффективным дивизором $K_{\widetilde{X}}+\tilde{\Delta}+\widetilde{\Upsilon} \equiv-\widetilde{S}$. Так как дивизор $K_{\widehat{X}}+\Delta+S+\Upsilon$ численно тривиален, то для любого дивизора $E$ поля $K(X)$ имеем

$$
a(E, \Delta+S+\Upsilon)=a(E, \tilde{\Delta}+\widetilde{S}+\widetilde{\Upsilon})
$$

(cp. [11, предложение 3.10]). Это показывает, что $(\widetilde{X}, \tilde{\Delta}+\widetilde{S}+\widetilde{\Upsilon})-$ чисто логтерминальная пара. Далее, по лемме $3.14 p(\widetilde{S})=o$. Так как дивизор $-\widetilde{S}$ численно эффективен над $Z$, то легко видеть, что $\widetilde{S}$ совпадает со слоем над $o$. По построению

$$
n\left(K_{\widetilde{S}}+\operatorname{Diff}_{\widetilde{S}}(\tilde{\Delta}+\widetilde{\Upsilon})\right) \sim 0
$$

и дивизор $K_{\widetilde{S}}+\operatorname{Diff}_{\widetilde{S}}(\tilde{\Delta}+\widetilde{\Upsilon})$ логтерминален по Кавамате (по формуле присоединения [13, теорема 17.6$])$. Поэтому

$$
\operatorname{totaldiscr}\left(\widetilde{S}, \operatorname{Diff}_{\widetilde{S}}(\tilde{\Delta}+\widetilde{\Upsilon})\right) \geqslant-1+1 / n, \quad n \leqslant N_{2} .
$$

Очевидно, что $\operatorname{Diff}_{\widetilde{S}}(\tilde{\Delta}+\widetilde{\Upsilon}) \neq 0$. Согласно [2] многообразие $\widetilde{S}$ принадлежит конечному числу алгебраических семейств. Таким образом, можно предположить, что число $\rho(\widetilde{S})$ ограничено константой Const $(\varepsilon)$.

Далее рассмотрим точную последовательность

$$
0 \longrightarrow \mathbb{Z} \longrightarrow \mathscr{O}_{\widetilde{X}}^{\mathrm{an}} \stackrel{\exp }{\longrightarrow} \mathscr{O}_{\widetilde{X}}^{\mathrm{an}} * \longrightarrow 0
$$

По теореме Каваматы-Фивега об обрашении в нуль $R^{i} f^{*} \mathscr{O}_{\widetilde{X}}^{\text {an }}=0, i>0$. Следовательно, $\operatorname{Pic}^{\text {an }}(\widetilde{X})=H^{2}(\widetilde{X}, \mathbb{Z})$. Аналогично, $H^{2}(\widetilde{S}, \mathbb{Z})=\operatorname{Pic}(\widetilde{S})$. Так как $\widetilde{S}=p^{-1}(o)$ - топологический ретракт $\widetilde{X}$, то $H^{2}(\widetilde{X}, \mathbb{Z})=H^{2}(\widetilde{S}, \mathbb{Z})$. Следовательно, число $\rho^{\text {an }}(\widetilde{X})$ ограничено и ограничено также $\rho^{\text {an }}(\widehat{X})$ (так как $\widehat{X} \rightarrow \widetilde{X}-$ последовательность флипов). Это доказывает утверждение 1$)$. Чтобы доказать 2$)$, можно использовать то, что $\rho^{\text {an }}(X / Z)$ равно числу компонент слоя $f^{-1}($ o) (по аргументации, приведенной выше; см. [15, следствие 1.3]).

СлеДСтвиЕ 4.8. Фиксируем $\varepsilon>0$. Пусть $(Z \ni о, D)$ - трехмерная исключительная логтерминальная по Кавамате особенность такая, что

$$
\text { totaldiscr }(X, D)>-1+\varepsilon
$$

и $D \in \Phi_{\mathbf{m}}^{3}$. Тогда для ее $\mathbb{Q}$-факториализачии $f: X \rightarrow Z$ имеем:

1) числа $\rho(X / Z)$ и $\rho^{\text {an }}(X / Z)$ ограничены Const $(\varepsilon)$;

2) число компонент $f^{-1}\left(\right.$ o) ограничено $\operatorname{Const}^{\prime}(\varepsilon)$.

Заметим, что для неисключительных малых стягивания число компонент слоя не ограничено даже в терминальном случае (см. [12, пример 13.7]). Мы предложим пример флоп-стягивания такого, как в следствии 4.7 . 
ПримеР 4.9. Пусть $(Z \ni o)$ - трехмерная гиперповерхностная особенность, заданная уравнением

$$
x_{1}^{3}+x_{2}^{3}+x_{3}^{5}+x_{4}^{5}=0
$$

в $\mathbb{C}^{4}$. Согласно [7] эта особенность исключительна (и канонична). Легко видеть также, что особенность не $\mathbb{Q}$-факториальна. Пусть $f: X \rightarrow Z$ - $\mathbb{Q}$-факториализация [13, теорема 6.11.1]. По лемме 2.3 пара $(X / Z \ni o, 0)$ исключительна. Следовательно, она удовлетворяет условиям следствия 4.7 (с $D=0)$.

Много примеров исключительных особенностей может быть найдено в работах [14] и [7]. Наконец, мы предложим пример исключительного стягивания Фано $f: X \rightarrow Z, \operatorname{dim}(X)>\operatorname{dim}(Z)$.

ПримеР 4.10 [20, теорема 7.1.12]. Рассмотрим поверхность $\mathbb{P}^{1} \times \mathbb{C}^{1}$ и раздуем точки на слое ее проекции $\mathbb{P}^{1} \times \mathbb{C}^{1} \rightarrow \mathbb{C}^{1}$ так, чтобы получить расслоение $f^{\text {min }}: X^{\text {min }} \rightarrow \mathbb{C}^{1}$ с центральным слоем, имеющим следующий двойственный граф:

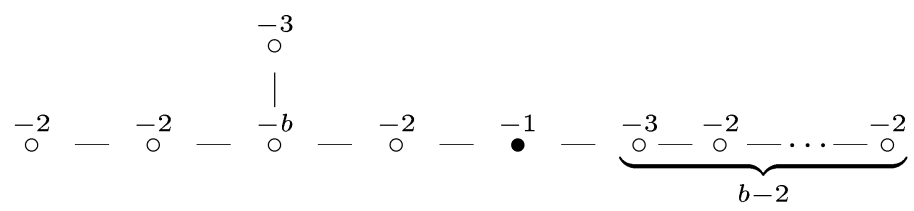

где $b \geqslant 2$. Далее стянем кривые, соответствуюшие белым вершинам. Мы получим экстремальное стягивание $f: X \rightarrow \mathbb{C}^{1}$ с двумя логтерминальными точками. Канонический дивизор $K_{X} 3$-дополняем, но не 1- или 2-дополняем [20, теорема 7.1.12]. Следовательно, стягивание $f$ исключительно.

\section{§ 5. Глобальный случай}

В этом параграфе модифицируем теорему 3.1 для глобального случая. В отличие от локального случая, здесь мы вынуждены предположить также существование гранищы с довольно "плохими" особенностями. Теорема 5.1 представляет собой частный случай гипотезы 1.9.

ТЕорема 5.1 (глобальньй случай). Пусть $(X, D)-d$-мерное логмногообразие глобального типа такое, что:

1) пара $(X, D)$ логтерминальна по Кавамате;

2) дивизор $-(K+D)$ численно әффективен и оббемен;

3) $D \in \Phi$, әде $\Phi=\Phi_{\mathbf{m}}^{d}$ или $\Phi=\Phi_{\mathbf{s m}}$.

Допустим, что имеется граница $D^{b}$ такая, что:

4) пара $K+D+D^{b}$ не логтерминальна по Кавамате;

5) дивизор $-\left(K+D+D^{b}\right)$ численно әффективен и обгемен.

Предположим также, что верна логПММ для размерности $d$. Тогда существует не логтерминальное по Кавамате $n$-дополнение дивизора $K+D$ $\partial \Omega$ s $n \in N_{d-1}(\Phi)$. 
ДокаЗАТельСТво. Сначала заменим $X$ на его $\mathbb{Q}$-факториализацию. Тогда, как и в лемме 3.3 , мы рассмотрим границу $D^{\mho} \geqslant 0$ такую, что дивизор $-(K+D+$ $D^{\mathrm{b}}+D^{\mho}$ ) обилен (но $K+D+D^{\mathrm{b}}+D^{\mho}$ необязательно логканоничен). Далее, найдем $t \in \mathbb{Q}$ такое, что пара $\left(X, D+t\left(D^{b}+D^{\mho}\right)\right)$ логканонична, но не логтерминальна по Кавамате (т.е. $t$ - логканонической порог $c\left(X, D, D^{b}+D^{\mho}\right)$ ). Положим $D^{\nabla}=$ $t\left(D^{b}+D^{\mho}\right)$.

Далее доказательство протекает аналогично доказательству теоремы 3.1.

СлеДСтвИЕ 5.2 (ср. [24, следствие 2.8]). Пусть $(X, D)$ - $d$-мерное логмногообразие глобального типа такое, что:

1) пара $(X, D)$ логтерминальна по Кавамате;

2) дивизор $-(K+D)$ численно эффективен и обвемен;

3) $D \in \Phi$, əде $\Phi=\Phi_{\mathrm{m}}^{d}$ или $\Phi=\Phi_{\mathrm{sm}}$;

4) $(K+D)^{d}>d^{d}$.

Предположим, что верна логПММ для размерности d. Тогда существует не логтерминальное по Кавамате $n$-дополнение $K+D$, где $n \in N_{d-1}(\Phi)$.

ДоказАтельство. Гранища $D^{\text {b }}$ такая, как в теореме 5.1, существует по теореме Римана-Роха (см., например, [11, лемма 6.7.1]).

Много примеров исключительных логповерхностей дель Пецщо могут быть найдены в работах [24], [1], [9] и [20].

\section{§6. Добавление}

В этом параграфе мы представим два полезных для приложений свойства дополнений. Будет использоваться определение 1.1 для случая, когда $D$ - субграница, т.е. $\mathbb{Q}$-дивизор (необязательно эффективньй) с коэффициентами $d_{i} \leqslant 1$.

ПредЛОЖЕНИЕ 6.1 [24, лемма 2.13]. Фиксируем $n \in \mathbb{N}$. Пусть $f: Y \rightarrow X-$ бирациональное стягивание, и пусть $D$ - субграница на $Y$ такая, что:

1) дивизор $K_{Y}+D$ численно эффективен над $X$;

2) $f(D)=\sum d_{i} f\left(D_{i}\right)$ - граница, чви коэффициенты удовлетворяют неравенству

$$
\left\lfloor(n+1) d_{i}\right\rfloor \geqslant n d_{i} .
$$

Предположим, что дивизор $K_{X}+f(D)$ n-дополняем. Тогда и дивизор $K_{Y}+D$ также п-дополняем.

ДоказательСтво. Рассмотрим крепантньй прообраз

$$
K_{Y}+D^{\prime}=f^{*}\left(K_{X}+f(D)^{+}\right), \quad f_{*} D^{\prime}=D
$$

Запишем $D^{\prime}=S^{\prime}+B^{\prime}$, где дивизор $S^{\prime}$ приведен, дивизоры $S^{\prime}$ и $B^{\prime}$ не имеют общих компонент и $\left\lfloor B^{\prime}\right\rfloor \leqslant 0$. Мы утверждаем, что $K_{Y}+D^{\prime}-n$-дополнение дивизора $K_{Y}+D$. Единственное, что нужно проверить, - это неравенство

$$
n B^{\prime} \geqslant\lfloor(n+1)\{D\}\rfloor .
$$


Из условия 2) имеем $f(D)^{+} \geqslant f(D)$. Этот дает нам, что $D^{\prime} \geqslant D$ (так как $D-D^{\prime}$ $f$-численно эффективен; см. $\left[23\right.$, п. 1.1]). Наконец, так как $n D^{\prime}$ - цельй дивизор, то имеет место неравенство

$$
n D^{\prime} \geqslant n S^{\prime}+\left\lfloor(n+1) B^{\prime}\right\rfloor \geqslant n\lfloor D\rfloor+\lfloor(n+1)\{D\}\rfloor .
$$

Следующее утверждение является усовершенствованием техники доказательств теоремы 5.6 из [23] и теоремы 19.6 из [13].

ПредлОЖЕНИЕ 6.2 [19]. Фиксируем $n \in \mathbb{N}$. Пусть $(X / Z \ni о, D)$ - логмногообразие и $S:=\lfloor D\rfloor, \quad B:=\{D\}$. Предположим, что:

1) пара $(X, D)$ чисто логтерминальна;

2) дивизор $-\left(K_{X}+D\right)$ иисленно эффективен и обвемен над $Z$;

3) $S \neq 0$ вблизи слоя $f^{-1}($ o);

4) коэффициенты дивизора $D=\sum d_{i} D_{i}$ удовлетворяют неравенству

$$
\left\lfloor(n+1) d_{i}\right\rfloor \geqslant n d_{i} .
$$

Далее, допустим, что вблизи $f^{-1}(o) \cap S$ существует $n$-дополнение $K_{S}+$ $\operatorname{Diff}_{S}(B)^{+}$логдивизора $K_{S}+\operatorname{Diff}_{S}(B)$. Тогда вблизи $f^{-1}($ o) существует $n$-дополнение $K_{X}+S+B^{+}$логдивизора $K_{X}+S+B$ mакое, что $\operatorname{Diff}_{S}(B)^{+}=$ $\operatorname{Diff}_{S}\left(B^{+}\right)$.

ДокАЗАТЕльСТво. Пусть $g: Y \rightarrow X-$ логразрешение. Запишем

$$
K_{Y}+S_{Y}+A=g^{*}\left(K_{X}+S+B\right),
$$

где $S_{Y}$ - собственный прообраз $S$ на $Y$ и $\lfloor A\rfloor \leqslant 0$. По формуле присоединения [13, теорема 17.6] дивизор $S$ нормален, а пара $\left(S, \operatorname{Diff}_{S}(B)\right)$ чисто логтерминальна. В частности, $g_{S}: S_{Y} \rightarrow S$ - бирациональное стягивание. Поэтому

$$
K_{S_{Y}}+\operatorname{Diff}_{S_{Y}}(A)=g_{S}^{*}\left(K_{S}+\operatorname{Diff}_{S}(B)\right) .
$$

Заметим, что $\operatorname{Diff}_{S_{Y}}(A)=\left.A\right|_{S_{Y}}$ (так как многообразие $Y$ неособо). Легко показать (см. $\left[20\right.$, п. 4.2]), что коэффициенты $\operatorname{Diff}_{S}(B)$ удовлетворяют неравенству (6.1). Таким образом, мы можем применить предложение 6.1 к $g_{S}$. Получаем $n$-дополнение $K_{S_{Y}}+\operatorname{Diff}_{S_{Y}}(A)^{+}$дивизора $K_{S_{Y}}+\operatorname{Diff}_{S_{Y}}(A)$. В частности, согласно (1.1) сушествует дивизор

$$
\Theta \in\left|-n K_{S_{Y}}-\left\lfloor(n+1) \operatorname{Diff}_{S_{Y}}(A)\right\rfloor\right|
$$

такой, что

$$
n \operatorname{Diff}_{S_{Y}}(A)^{+}=\left\lfloor(n+1) \operatorname{Diff}_{S_{Y}}(A)\right\rfloor+\Theta .
$$

По теореме Каваматы-Фивега об обрашении в нуль имеем

$$
\begin{aligned}
R^{1} h_{*}\left(\mathscr{O}_{Y}\left(-n K_{Y}-(n+1) S_{Y}-\lfloor(n+1) A\rfloor\right)\right) \\
\quad=R^{1} h_{*}\left(\mathscr{O}_{Y}\left(K_{Y}+\left\lceil-(n+1)\left(K_{Y}+S_{Y}+A\right)\right\rceil\right)\right)=0 .
\end{aligned}
$$


Из точной последовательности

$$
\begin{aligned}
0 & \longrightarrow \mathscr{O}_{Y}\left(-n K_{Y}-(n+1) S_{Y}-\lfloor(n+1) A\rfloor\right) \\
& \longrightarrow \mathscr{O}_{Y}\left(-n K_{Y}-n S_{Y}-\lfloor(n+1) A\rfloor\right) \\
& \longrightarrow \mathscr{O}_{S_{Y}}\left(-n K_{S_{Y}}-\left.\lfloor(n+1) A\rfloor\right|_{S_{Y}}\right) \longrightarrow 0
\end{aligned}
$$

мы получаем сюръективность ограничения

$$
\begin{aligned}
H^{0}\left(Y, \mathscr{O}_{Y}\left(-n K_{Y}-n S_{Y}-\lfloor(n+1) A\rfloor\right)\right) \longrightarrow \\
\quad \longrightarrow H^{0}\left(S_{Y}, \mathscr{O}_{S_{Y}}\left(-n K_{S_{Y}}-\left.\lfloor(n+1) A\rfloor\right|_{S_{Y}}\right)\right)
\end{aligned}
$$

Поэтому существует дивизор

$$
\Xi \in\left|-n K_{Y}-n S_{Y}-\lfloor(n+1) A\rfloor\right|
$$

такой, что $\left.\Xi\right|_{S_{Y}}=\Theta$. Положим

$$
A^{+}:=\frac{1}{n}(\lfloor(n+1) A\rfloor+\Xi) .
$$

Тогда $n\left(K_{Y}+S_{Y}+A^{+}\right) \sim 0$ и $\left.\left(K_{Y}+S_{Y}+A^{+}\right)\right|_{S_{Y}}=K_{S_{Y}}+\operatorname{Diff}_{S_{Y}}(A)^{+}$.

Заметим, что обрашение присоединения на $Y$ неприменимо потому, что $A^{+}$может иметь отрицательные коэффициенты. Чтобы исправить ситуацию, положим $B^{+}:=g_{*} A^{+}$. Снова имеем $n\left(K_{X}+S+B^{+}\right) \sim 0$ и

$$
\left.\left(K_{X}+S+B^{+}\right)\right|_{S}=K_{S}+\operatorname{Diff}_{S}(B)^{+} .
$$

Покажем только то, что дивизор $K_{X}+S+B^{+}$логканоничен. Предположим противное. Тогда пара $\left(X, S+B+\alpha\left(B^{+}-B\right)\right)$ также не логканонична для некоторого $\alpha<1$. Ясно, что дивизор $-\left(K_{X}+S+B+\alpha\left(B^{+}-B\right)\right)$ численно эффективен и объемен над $Z$. По обращению присоединения [13, теорема 17.6] пара $\left(X, S+B+\alpha\left(B^{+}-B\right)\right)$ чисто логтерминальна вблизи $S \cap f^{-1}(o)$. Следовательно, $L C S\left(X, B+\alpha\left(B^{+}-B\right)\right)=S$ вблизи $S \cap f^{-1}(o)$. С другой стороны, по лемме о связности [13, теорема 17.4] множество $L C S\left(X, B+\alpha\left(B^{+}-B\right)\right)$ связно вблизи $f^{-1}(o)$. Таким образом, пара $\left(X, S+B+\alpha\left(B^{+}-B\right)\right)$ чисто логтерминальна. Последнее противоречие доказьвает предложение.

\section{Список литературы}

1. Abe T. Classification of Exceptional Complements: Elliptic Curve Case. e-print alg-geom/ 9711029.

2. Alexeev V. Boundedness and $K^{2}$ for $\log$ surfaces // Int. J. Math. 1994. V. 5. P. 779-810.

3. Borisov A. A. Boundedness theorem for Fano log-threefolds // J. Algebraic Geom. 1996. V. 5. P. 119-133.

4. Борисов А. А., Борисов Л. А. Особые торические многообразия Фано // Матем. сб. 1993. T. 75. C. 277-283.

5. Fujino O. Abandance theorem for semi log canonical threefolds // Duke Math. J. 2000. V. 102. № 3. P. 513-532.

6. Ishii $S$. The quotients of log-canonical singularities by finite groups // Singularities. Sapporo, 1998; // Adv. Stud. Pure Math. 2000. V. 29. P. 135-161. 
7. Ishii S., Prokhorov Yu. G. Hypersurface exceptional singularities // International J. Math. 2001. V. 12. № 5. P. 1-27; e-print math.AG/9910123.

8. Kawamata Y., Matsuda K., Matsuki K. Introduction to the minimal model program // Adv. Stud. Pure Math. 1987. V. 10. P. 283-360.

9. Keel S., McKernan J. Rational curves on quasi-projective surfaces // Memoirs AMS. 1999. V. 140. № 669 .

10. Kollár J. Log surfaces of general type; some conjectures // Contemporary Math. AMS. 1994. V. 162. P. 261-275.

11. Kollár J. Singularities of pairs // Proc. Symp. Pure Math. 1995. V. 62. P. 221-287.

12. Kollár J., Mori S. Classification of three dimensional flips // J. Amer. Math. Soc. 1995. V. 5. P. 533-703.

13. Kollár J. et al. Flips and abundance for algebraic threefolds // A summer seminar at the University of Utah. Salt Lake City. 1991. V. 211. Astérisque, 1992.

14. Markushevich D., Prokhorov Yu. G. Exceptional quotient singularities // Amer. J. Math. 1999. V. 121. P. 1179-1189.

15. Mori S. Flip theorem and the existence of minimal models for 3-folds // J. Amer. Math. Soc. 1988. V. 1. P. 117-253.

16. Никулин В. В. Поверхности дель Пеццо с логтерминальными особенностями. I // Матем. сб. 1989. Т. 180. С. 226-243.

17. Никулин В. В. Поверхности дель Пеццо с логтерминальными особенностями. III // Изв. АН СССР. Сер. матем. 1989. Т. 53. №6. С. 1316-1334.

18. Prokhorov Yu. G. Blow-ups of canonical singularities // "Algebra" - Proc. Internat. Algebraic Conf., Moscow, Russia, May 25-30, 1998 / Ed. Yu. Bahturin. Berlin: Walter der Greither Publ., 2000. P. 301-317.

19. Prokhorov Yu. G. Boundedness of nonbirational extremal contractions // Internat. J. Math. 2000. V. 11. P. 393-411.

20. Prokhorov Yu. G. Lectures on complements on log surfaces // MSJ Memoirs. V. 10. Math. Soc. of Japan, 2001.

21. Прохоров Ю.Г. Ограниченность исключительных факторособенностей // Матем. заметки. 2000. Т. 68. № 5. С. 786-789.

22. Shepherd-Barron N.I., Wilson P. M. H. Singular threefolds with numerically trivial first and second Chern classes // J. Algebraic Geom. 1994. V. 3. P. 265-281.

23. Шокуров В. В. Трехмерные логперестройки // Изв. АН СССР. Сер. матем. 1993. Т. 40. C. 93-202.

24. Shokurov V. V. Complements on surfaces // J. Math. Sci. 2000. V. 102. № 2. P. 3876-3932.

25. Shokurov V. V. 3-fold log models // J. Math. Sci. 1996. V. 81. P. 2667-2699.

26. Shokurov V. V. Pl flips. Preprint/Baltimore-Moscow, 2000.

Ю. Г. Прохоров: Московский государственный университет

им. М. В. Ломоносова;

Поступило в редакцию

e-mail: prokhoro@mech.math.msu.su

3.X. 2000

В. В. Шокуров: The Johns Hopkins University,

Department of Mathematics, Baltimore, Maryland, USA

e-mail: shokurov@math.jhu.edu 